\title{
Duplicative search*
}

\author{
Alexander Matros ${ }^{\dagger} \quad$ Vladimir Smirnov ${ }^{\ddagger}$
}

June 23, 2016

\begin{abstract}
In this paper we examine the dynamic search of two rivals looking for a prize of known value that is hidden in an unknown location, modeled as search for treasure on an island. In every period, the players choose how much to search of the previously unsearched portion of the island in a winner-takes-all contest. If the players cannot coordinate so as to avoid searching the same locations, the unique equilibrium involves complete dissipation of rents. On the other hand, if the players have some (even limited) ability to coordinate so as to avoid duplicative search and the search area is sufficiently small, there is a unique equilibrium in which the full area is searched and each player earns a positive expected return.

Keywords: R\&D, search, duplication, uncertainty, coordination.

JEL classifications: D21, O32.
\end{abstract}

\section{Introduction}

Consider pharmaceutical firms competing in a race to discover a new drug, paparazzi searching luxury hotels for a visiting movie star, bounty hunters pursuing a fugitive, or researchers looking for solutions to the six Millennium Prize Problems in mathematics. All of these scenarios have attributes of a general problem, namely, a treasure hunt for a prize of known value in which there is uncertainty regarding the cost of search. Critically, in each of these cases, a searching party's incentive changes dynamically as search progresses. For instance, a paparazzi's incentive to search depends on the number of remaining unsearched hotels. Similarly, pharmaceutical companies strategically adjust their effort as research into

*We would like to thank Murali Agastya, Suren Basov, Jay Pil Choi, Vincent P. Crawford, Peter Exterkate, Simon Kwok, Jordi McKenzie, Mark Melatos, Jonathan Newton, Michael Paton, Suraj Prasad, Nicolas de Roos, John Romalis, Robert Rothschild, Abhijit Sengupta, Kunal Sengupta, Rami Tabri, Russell Toth, Andrew Wait, Don Wright, Charles Zworestine, participants at the 9th Annual International Industrial Organization Conference in Boston and, in particular, the Advisory Editor and two anonymous referees for their helpful comments.

${ }^{\dagger}$ Moore School of Business, University of South Carolina and Lancaster University Management School; alexander.matros@gmail.com.

${ }^{\ddagger}$ School of Economics, University of Sydney; vladimir.smirnov@sydney.edu.au. 
a new medication progresses. The dynamics - and changing nature of search - is critical in each of these problems. In this paper we analyze a dynamic non-stationary search model for a known prize, hidden in an unknown location.

To do this, we develop a model in which two players undertake costly search for a treasure of known value that is hidden somewhere on an island of a given area. We assume that there is an equal probability that the treasure will be located at any given point on the island. In each period, both players make their search decisions simultaneously. Once the treasure is found the game ends. If the treasure is not discovered in any given period, the game continues. In the next period, each player observes the locations that have previously been searched. They then decide how much of the remaining area they wish to investigate. If the treasure is simultaneously discovered - this happens if the two players successfully search the same part of the island - both players incur their individual costs but the treasure is destroyed. If a player discovers the treasure on their own, they receive the full value of the treasure, while both players incur their search costs.

First, we consider the case when the duopolists are unable to coordinate to avoid searching the same location. Given its dynamic nature, we analyze a game in which each state is described by the remaining unsearched area. Following Maskin and Tirole (1988), we restrict our attention to symmetric Markov perfect equilibria (SMPE). ${ }^{1}$ With uncoordinated search, there is a unique equilibrium in which the potential value of the prize is completely dissipated by excessive search. To provide some intuition for this dissipation result, consider the special case when there is only one possible search period. As in the standard price-setting Bertrand game, each player's payoff maximizing search behavior drives their rival's expected return to zero. The generalized game with more than one possible search period involves the same essential tradeoff, ensuring complete dissipation. This result has far-reaching economic implications for those participating in a treasure hunt, be they pharmaceutical firms, paparazzi, bounty hunters or pirates.

Second, if the players have some ability to coordinate search so as to avoid duplication, the equilibrium in which all rents are dissipated no longer holds; that is, positive expected returns are feasible even when the players can only partially coordinate their search activities. The possibility of coordination may arise between the players through observation. For example it might be evident where miners are moving their equipment, or the direction that a paparazzo is heading; a bounty hunter might have better contacts in one area than another, or might only have a permit to work in a particular jurisdiction. Coordination could also come about through some form of commitment by a player. For example, the sort of experts a research department hires provides a (partial) commitment to the direction of their search. Similarly, pharmaceutical companies might need to make public announcements (in broad terms) about their upcoming clinical trials, and their existing patents will highlight the most obvious place a firm will start looking for the next generation of drug. These coordination devices are, of course, only partial - these commitments can, to a greater or lesser extent, be unwound. Importantly, our modeling suggests that

\footnotetext{
${ }^{1}$ Imposing Markov perfection not only makes our analysis simpler, while still being consistent with rationality, but it also makes our results directly comparable to those in the previous literature. See Maskin and Tirole (1988), Bhaskar et al. (2012) and Battaglini et al. (2014) for a general discussion of when the use of SMPE is appropriate.
} 
however imperfect, the possibility of some coordination creates an environment in which complete dissipation of rents is not possible. This suggests that a third party (government, industry association and so on) could improve on the decentralized market outcome, even if its regulatory technology is quite imperfect.

Duplication with independent search also arises in the model of Fershtman and Rubinstein (1997). They consider a model in which two players search for a single hidden treasure in one of a given set of labeled boxes. First, each player chooses how many costly search units they wish to utilize. Second, each player chooses a random search strategy with which to examine the boxes, where only one box can be examined in any given period by each search unit. While each player knows the total number of boxes their rival can search, all research findings are private information. Their model gives rise to some complementary results to ours. For example, there can be search duplication in equilibrium, when players search previously opened boxes that are empty. Almost the opposite type of coordination failure is also possible: with an asymmetric probability that various boxes contain the treasure, sometimes neither player searches the highest probability box first. The first of these failures does not occur in our model as previous search is observable to all. The second outcome is not possible here either due to our focus on pure strategies. Perhaps the most important similarity is that with independent search rents are completely dissipated in both models. The intuition is also similar: in Fershtman and Rubinstein (1997) each player chooses the number of search units in the first stage of the game such that the other player has a zero expected return; in our paper each searcher chooses an area to investigate each period that drives the other player's return to zero.

Duplication and coordination by duopolists in a dynamic R\&D search game is the focus of Chatterjee and Evans (2004). In their model, in each period two competing firms simultaneously choose to research one of two projects, where only one project will eventually be successful (given it has been investigated long enough). As in our model, previous investment is observable and it is common knowledge. They assume, however, that firms can coordinate their search in any given period. In Chatterjee and Evans (2004), only under certain conditions will search be efficient. More typically, the outcome will be inefficient due to too much duplication (reminiscent of our dissipation result above) or too much diversification (in which the firms have too much incentive to investigate separate projects). In particular, if the costs of the two projects are unequal, then there is an equilibrium in which the high-cost project is searched too often, relative to the socially efficient choice. One important difference between their model and ours is that per-period search costs are exogenous in their model, whereas search costs in our model endogenously depend on the search undertaken in any given period. Critically, search endogeneity and lack of coordination in our model leads to a complete dissipation of expected rents, which is not observed in Chatterjee and Evans (2004).

There is a literature that models research contests as rank-order tournaments. In contrast to our paper, this literature analyzes the situation in which there are multiple potential innovations that compete against each other. Some examples recently discussed in the literature include: a 1992 refrigerator competition (see Taylor, 1995), an 1829 steam locomotion tournament (see Fullerton and McAfee, 1999), a 1714 British contest for a method of determining longitude at sea (see Che and Gale, 2003). Within this framework, 
both Erat and Krishnan (2012) and Konrad (2014) consider the issue of duplication. In Erat and Krishnan (2012), firms simultaneously select which area (or avenue of research) of many clearly delineated areas to search. Each avenue has its own value. In equilibrium, the expected return from choosing any given area is equalized, with more firms opting to search avenues with a higher value. Coordination is facilitated in Erat and Krishnan (2012) by the ability to commit to only search in a particular avenue. As in our model with partial search coordination, and in a similar manner to Chatterjee and Evans (2004), while there is duplication, not all rents are dissipated. Konrad (2014) also considers a model in which a firm must choose a specific avenue of research, where these avenues do not overlap. Importantly, if two or more firms choose the same avenue, any rents generated are dissipated, as in Bertrand competition. Again, it is the ability of firms to commit to search only one avenue that facilitates coordination. In equilibrium there is no duplication, as no two firms choose the same avenue of research. In our model, a parallel result arises when duopolists can perfectly coordinate their search patterns.

In many ways, our paper synthesizes the existing literature, from the model with independent search of Fershtman and Rubinstein (1997), to the perfect coordination case in Konrad (2014), to the partial coordination search models in between of Chatterjee and Evans (2004) and Erat and Krishnan (2012). One important difference between our paper and the existing literature is that in our model firms are choosing how much to search every period (allowing for search dynamics). On the other hand, Chatterjee and Evans (2004), Erat and Krishnan (2012) and Konrad (2014) effectively assume that the amount of search is given exogenously, while in Fershtman and Rubinstein (1997) an equivalent choice is made only once at the beginning at the game.

Our work extends the previous research on private-good search. ${ }^{2}$ These models mostly deal with situations that are either static or involve complete information. ${ }^{3}$ One of the few papers that considers the dynamics of investment within the private-good framework is Reinganum (1981), who assumes that the success function is exponential and the environment is stationary. ${ }^{4}$ As a result, equilibrium strategies can be represented as functions of time only, which greatly simplifies the analysis. ${ }^{5}$ The memorylessness assumption, however, is often not satisfactory; for example, when the attractive search domain, while potentially large, is finite. As noted above, one of the contributions of this paper is finding equilibria in a truly dynamic search model, in that players' choices regarding effort depend on the remaining unsearched island size. Our framework allows for another important difference with the existing literature. With a uniform distribution, investing always becomes more attractive over time, ensuring that once firms start investing, they will continue until the

\footnotetext{
${ }^{2}$ There is also a large literature on investment dynamics in relation to public goods; for instance, Admati and Perry (1991), Marx and Matthews (2000), Lockwood and Thomas (2002), Compte and Jehiel (2004), Yildirim (2004, 2006), Bonatti and Hörner (2011), Matthews (2013), Battaglini et al. (2014) and Georgiadis (2015).

${ }^{3}$ See, for example, Loury (1979), Dasgupta and Stiglitz (1980a,b) and Lee and Wilde (1980), and Reinganum (1989) and Long (2010) for a survey of the literature.

${ }^{4}$ In the context of our model, this would be equivalent to assuming that the present value of the treasure is constant.

${ }^{5}$ Reinganum (1982) uses a similar framework to show how the availability of patent protection can accelerate development of the innovation.
} 
treasure is discovered (or the search area is exhausted). This is very different to the outcome with an exponential distribution typically used in the literature where, if there is a positive probability that the project is unsuccessful, investing becomes less attractive over time and it is possible that the project could be suspended.

\section{The Model}

In the model, two players (firms) search over an infinite horizon for a treasure hidden somewhere on an island of size $x_{1}>0$. The treasure has the same value $R>0$ for both players $^{6}$, and there is an equal probability that the treasure will be located at any given point on the island. ${ }^{7}$ Payoffs are discounted at a common factor $\delta$.

Each period $t$ comprises the following sequence of events. First, both players learn the size and location of all prior searches. ${ }^{8}$ As a convention, we use the term 'area' to mean the size of land searched and the term 'region' to mean the location of the search. Let $x_{t}$ be the unsearched area of the island at the beginning of period $t$. Second, each player $i$ simultaneously plans an area $I_{i, t} \in\left[0, x_{t}\right]$ to search at a cost of $c I_{i, t}$, where $c>0 .{ }^{9}$ The regions searched by the players are partially coordinated according to a process discussed below. Finally, search plans are implemented. If the treasure is found, the game ends. If both players search the same region and find the treasure simultaneously, each of them incurs costs, but the treasure is destroyed. ${ }^{10}$

Suppose that player $i$ searches an area $I_{i, t}$ in period $t$, for $i=1,2$. If the region of search is chosen uniformly at random, the probability that $i$ searches a specific point on the island is $I_{i, t} / x_{t}$. If both players were to choose their search region independently, then the probability that both players search this particular point would be $I_{1, t} I_{2, t} / x_{t}^{2}$, and the area of duplicated search would be $I_{1, t} I_{2, t} / x_{t} \cdot{ }^{11}$

Under our specification of partial coordination, the extent of duplication is given by $(1-\alpha) I_{1, t} I_{2, t} / x_{t}$, where the parameter $\alpha \in[0,1]$ describes the extent of coordination. If

\footnotetext{
${ }^{6}$ As players are risk neutral, in the case of random returns $R$ could be replaced by $E[R]$, requiring no other changes to the model.

${ }^{7}$ In Appendix B we consider the case when the distribution is non-uniform.

${ }^{8}$ Using the terminology of Mailath and Samuelson (2006), we consider a stochastic game with perfect monitoring in a dynamic setting.

${ }^{9}$ Non-linear costs are discussed in Section 4.

${ }^{10}$ This assumption is standard in the R\&D literature. Intuitively, if several players discover the treasure simultaneously, fierce competition between them runs down the surplus to zero. A good example of such a situation for just two players is Lockheed and Douglas jet development in the 1960s. For more detail, see The Economist (1985); and Chatterjee and Evans (2004). Many examples of simultaneous discoveries in science can be found in Merton (1973).

${ }^{11}$ Independent search in each period of our dynamic game mimics the random search strategy of firms in the static model of Fershtman and Rubinstein (1997). We assume that a player's unsearched area (which we model as the $\left[0, x_{t}\right]$ segment) can be partitioned into a large number of equally-sized small segments. In this structure, from each player's perspective the treasure is equally likely to be located in any one of the segments. Given player $i$ chooses $I_{i, t}$, we assume he will randomly select a portion $I_{i, t} / x_{t}$ of all unsearched segments, where each of the unsearched segments is chosen with equal probability. For our analysis, the area of duplicated search is defined by its limit when the size of each segment goes to zero. See Appendix A for a detailed explanation.
} 
$\alpha=1$, search is perfectly coordinated and duplication is avoided; if $\alpha=0$, search is independent; and $\alpha \in(0,1)$ indicates partial coordination. ${ }^{12}$

If in any period $t$, player 1 searches $I_{1, t}$ and player 2 searches $I_{2, t}$, after accounting for duplication, the players search an area of

$$
J_{t}=\min \left\{x_{t}, I_{1, t}+I_{2, t}-(1-\alpha) I_{1, t} I_{2, t} / x_{t}\right\} .
$$

Player $i$ has a $\left(J_{t}-I_{j, t}\right) / x_{t}$ probability of finding the treasure alone and obtains the following expected payoff in period 1 :

$$
\frac{J_{t}-I_{j, t}}{x_{t}} R-c I_{i, t}
$$

The game ends with probability $J_{t} / x_{t}$ in period $t$. If the treasure is not found in period $t$, which happens with probability $1-J_{t} / x_{t}$, the unsearched area of the island shrinks to

$$
x_{t+1}=x_{t}-J_{t},
$$

and the game proceeds to period $t+1$.

Player $i$ 's strategy is an infinite sequence of functions specifying how much to search each period contingent upon any possible sequence of previous searches. We assume that at any stage, the entire history of past searches is common knowledge and can be summarized by the 'state', the current unsearched area. We will consider Markov strategies in which the past influences current play only through its effect on the current unsearched area. A pure Markov strategy for player $i$ is a time-invariant map $I_{i}: X \rightarrow X$, where $X=\left[0, x_{1}\right]$ and $I_{i}(x) \in[0, x]$. Note that for ease of exposition, when convenient we skip subscript $t$ and indicate that $I$ depends on the current unsearched area $x$; that is, $I_{i, t}=I_{i}(x)$. Also, when the meaning is clear, we skip subscript $i$ in describing the equilibrium search intensity and the value function.

We will restrict our attention to symmetric equilibria. Therefore, the solution concept we use is a symmetric Markov perfect equilibrium (SMPE). ${ }^{13}$ Moreover, we focus on nontrivial SMPE; that is, equilibria in which a positive amount of search occurs somewhere along the equilibrium path. We use the following approach to obtaining the SMPE. Player $i$ takes the state-contingent search plans of his rival $I_{j}(x)$ as given. Given this function, player $i$ solves a standard optimization problem and chooses his optimal search, $I_{i}(x)$. However, given that the function $I_{j}(x)$ is endogenous to the model, to obtain the symmetric equilibrium we need to find the function $I_{j}(x)$ such that $I_{i}(x) \equiv I_{j}(x)$, where $I_{i}(x)$ is the optimal search for player $i$ when he takes $I_{j}$ as given. ${ }^{14}$

\footnotetext{
${ }^{12}$ Different sources of partial coordination are possible. For example, the parameter $\alpha$ could describe the degree of flexibility of the players to adapt to the search plans of their rival. Alternatively, $\alpha$ could represent the proportion of information that is publicly available regarding each player's search, whereas $(1-\alpha)$ is the proportion that cannot be credibly revealed. Additional motivation for three different scenarios and micro-foundations for partial coordination are provided in Section 3.

${ }^{13}$ We define equilibria in terms of the policy functions $I_{1}$ and $I_{2}$, which describe the area but not the region of search. An equilibrium is therefore consistent with a multiplicity of outcomes relating to the region of search.

${ }^{14}$ We discuss both subgame perfect and asymmetric Markov perfect equilibria in Section 4.
} 
Note that when multiple SMPE exist, we focus on the Superior SMPE (SSMPE); that is, the SMPE with the highest total expected payoff. ${ }^{15}$

\section{Analysis of the model}

In this section we consider the equilibrium outcome of the model. To do this, let us first establish several preliminary results for the general model that we will then use in our subsequent analysis.

Player $i$ solves the following Bellman equation:

$$
V(x, c, R)=\max _{I_{i} \in[0, x]}\left\{-c I_{i}+\frac{J-I_{j}}{x} R+\delta\left(1-\frac{J}{x}\right) V(x-J, c, R)\right\},
$$

where $x$ is the part of the island which is still unsearched before the current period, $V(x, c, R)$ is the value function for each player (we use the symmetry assumption here), and $J$ is given by equation (1). The first term in equation (2) describes the player's costs of search in the current period. The second term is the player's expected value from finding the treasure alone in the current period. The last term is the player's expected value from future periods.

To simplify the analysis, we introduce the following lemma.

Lemma 1. The following identity always holds:

$$
V(x, c, R) / R=V(c x / R, 1,1) .
$$

Proof. See Appendix C.

An increase in the cost parameter $c$ in the original problem (2) is equivalent to an increase in the unsearched area size $x$. On the other hand, an increase in the treasure value $R$ has two effects; it decreases the unsearched area size $x$, but it also directly changes the value function. Effectively, the island size is proportional to $c$, inversely proportional to $R$, and payoffs are denominated in $R .^{16}$

Slightly abusing notation, rename $V(x)=V(x, 1,1)$. From Lemma 1 it follows that it is sufficient to solve equation (2) when $R=c=1$; that is,

$$
V(x)=\max _{I_{i} \in[0, x]}\left\{-I_{i}+\frac{J-I_{j}}{x}+\delta\left(1-\frac{J}{x}\right) V(x-J)\right\} .
$$

The following lemma allows us to distinguish between equilibria under independent and coordinated search.

\footnotetext{
${ }^{15}$ As argued by Fudenberg and Tirole (1985), if one equilibrium Pareto dominates all others, it is the most reasonable outcome to expect.

${ }^{16}$ For additional details and comparative statics, see our complementary paper Matros and Smirnov (2011).
} 
Lemma 2. Combined search $J$ described by equation (1) exhibits the following properties: i) If $\alpha=0, I_{1}<x$, and $I_{2}<x$, then $J<x$.

ii) If $\alpha \in(0,1]$, there exist $I_{1}<x$ and $I_{2}<x$ such that $J=x$.

Proof. See Appendix C.

Lemma 2 suggests that if $\alpha=0$, the entire island is searched in a given period only in the case that at least one player searches the entire island. As symmetry requires each player's search is the same, it is not possible for the players to search the entire island without destroying the treasure in a symmetric equilibrium. By contrast, with an element of coordination, it is possible for the players to complete the search without full dissipation of the treasure.

\subsection{Independent search $(\alpha=0)$}

Let us first consider the extreme case when players search independently. There are many situations when it is not possible to avoid duplication. For instance, the set of projects may have no obvious ordering and it may be very difficult to predict rival search plans; this means that the two players might end up simultaneously searching the same area. Duplication is more likely to arise when the players have no common background to rely on and players cannot observe the search of their rivals. With no common expectation of the search plans of any player, duplication could be unavoidable. ${ }^{17}$

Utilizing Lemma 2, when $\alpha=0$ equation (1) transforms to:

$$
J=I_{1}+I_{2}-I_{1} I_{2} / x .
$$

We analyse this case in the following proposition.

Proposition 1. If $\alpha=0$, in the unique $S M P E$

$$
I(x)=\max \left\{0, x-x^{2}\right\} \quad \text { and } \quad V(x) \equiv 0 .
$$

Proof. See Appendix C.

To get some intuition, let us consider the static problem where players can only search once and $\alpha=0$. Substituting for $J$, the problem in this case is

$$
V(x)=\max _{I_{i} \in[0, x]}\left\{-I_{i}+\frac{I_{i}}{x}\left(1-\frac{I_{j}}{x}\right)\right\}=\max _{I_{i} \in[0, x]}\left\{\frac{I_{i}\left(x-x^{2}-I_{j}\right)}{x^{2}}\right\} .
$$

If the search by its rival is $I_{j}<x-x^{2}$, it is a dominant strategy for player $i$ to choose $I_{i}(x)=x$. If, on the other hand, search by the other player is $I_{j}>x-x^{2}$, it is a dominant

\footnotetext{
${ }^{17}$ One example we have in mind is pharmaceutical companies trying to develop a new product, such as a sleeping pill. Given the secrecy surrounding work in these laboratories, it is not possible to guarantee that there is no duplication by rivals. For example, Eichler et al. (2013) discuss a documented case of duplication in medical research. Chatterjee and Evans (2004) also provide case-study evidence of simultaneous discovery leading to a dissipation of potential profits.
} 
strategy for player $i$ to opt $I_{i}(x)=0$. There is a unique symmetric equilibrium in which both players search $I(x)=\max \left\{0, x-x^{2}\right\}$ and each receives a value of zero. This result mimics the outcome in the standard Bertrand price-setting game in which the best response of each player ensures that all of the potential rents are dissipated in equilibrium.

In a similar manner, in the equilibrium of the dynamic game the island has no value to either player for any $x$. To see why this is an equilibrium, note that if $V(x)=0$ for all $x$, then (4) reduces to the static problem above, and the unique solution has a value of zero for both players. Because of the contraction property of the dynamic problem, this equilibrium is unique. The economic intuition for this equivalence between the static and dynamic problems is that there is effectively no connection between the periods; there is no benefit for a player to wait until next period, as in the next period players face exactly the same problem.

Note, not only do we have duplication in this extreme case with $\alpha=0$, the duplication here is so egregious that the whole value of the treasure is dissipated in equilibrium. Thus, independent search gives rise to an extreme example of the tragedy of the commons. A feature of equilibrium is that the island is never searched completely and search may continue for arbitrarily many periods.

\subsection{Coordination $\alpha \in(0,1]$}

We next consider the case when the parties have some ability to coordinate their search for the treasure. As mentioned above, there are times when parties can commit to a search pattern or to focus (or not) on a particular location. For example, pharmaceutical companies might have to lodge broad plans with the regulator regarding their upcoming clinical trials; these plans provide some (potentially imperfect) commitment to a given search strategy. Similarly, the hiring of experts in a given area of expertise, or the purchase of specific research hardware can also be observable commitment. Another possibility is that at the start of each period each player decides on the size of their research budget and specific search locations, but only the choice of research budget is observable to general public (possibly through required public announcements for a publicly listed firm). Given these budgetary commitments (which determine the area each player searches in that period), it is in the interests of the two rivals to coordinate in an attempt to avoid duplication. However, if it is too costly to adjust all search locations, only partial coordination is possible. ${ }^{18}$ In the model, $\alpha$ indicates the proportion of a player's search that can be adjusted, whereas $(1-\alpha)$ is the proportion of search that is sufficiently inflexible so that it cannot be adjusted. ${ }^{19}$ The generalization presented in this section allows us to capture both partial coordination of search $(0<\alpha<1)$ and perfect coordination $(\alpha=1)$.

To simplify the exposition of the model, it is convenient to introduce the following

\footnotetext{
${ }^{18}$ For example, we could assume that both parties reveal all locations of their search but only one party can reallocate some of the locations they initially planned to search. Note that the same micro-foundations as in the case of independent search apply here, where the area of duplication is scaled by $(1-\alpha)$.

${ }^{19}$ Chatterjee and Evans (2004) and Erat and Krishnan (2012) generate partial coordination by allowing perfect coordination with respect to project selection and independent search within a project.
} 
function:

$$
\Psi(x) \equiv x V(x)
$$

In terms of $\Psi(x)$, equation (4) can be rewritten as

$$
\Psi(x)=\max _{I_{i} \in[0, x]}\left\{J-I_{j}-x I_{i}+\delta \Psi(x-J)\right\} .
$$

Note that equation (7) resembles the problem of extraction of exhaustable resources under common access or, equivalently, a multi-player cake-eating problem. ${ }^{20}$

Before proceeding, we introduce the following operator $B$ defined by

$$
(B \Psi)(x) \equiv \max _{I_{i} \in[0, x]}\left\{J-I_{j}-x I_{i}+\delta \Psi(x-J)\right\} .
$$

This allows us to consider the following lemma.

Lemma 3. If $0<\alpha \leq 1$, there exists finite $T$ such that

(a) any non-trivial SMPE involves $J_{t}>0$ for $0 \leq t \leq T$, and $x_{T+1}=0$; and

(b) all SMPE can be obtained in T steps. Specifically,

$$
\Psi_{0} \equiv 0, \quad \Psi_{s} \equiv B \Psi_{s-1} \quad \text { for } \quad s=1,2, \ldots, T, \quad \text { and } \quad \Psi=\Psi_{T}
$$

Proof. See Appendix C.

Lemma 3 suggests that the treasure is found in finite time in any non-trivial SMPE. Discounting and coordination play important roles in this result. Discounting ensures that the value of indefinite search converges to zero. If search ever commences, it will be completed in finite time. To see the role of coordination, recall from Lemma 2 that only if search is coordinated is it possible to search the entire island and obtain positive value.

Furthermore, Lemma 3(b) reveals that the finite application of the operator $B$ represents an algorithm for solving the Bellman equation. From this, we derive the following proposition for when search is completed in one period.

Proposition 2. If $0<\alpha \leq 1, \forall x \leq \sqrt{\alpha}\left(1-\delta \frac{\sqrt{\alpha}}{1+\sqrt{\alpha}}\right)$ in the unique SMPE

$$
I(x)=\frac{x}{1+\sqrt{\alpha}} \quad \text { and } \quad V(x)=\frac{\sqrt{\alpha}-x}{1+\sqrt{\alpha}}>0 .
$$

Proof. See Appendix C.

This Proposition shows that when players have some non-zero ability to coordinate, the extreme dissipation result of Proposition 1 does not hold. Coordination allows for the existence of equilibria with some search duplication and a value function that is nonzero. Lemma 2 plays an integral role in this result. With coordination, it is possible for

\footnotetext{
${ }^{20}$ See for example Long (2011) for a recent survey of dynamics games in the economics of natural resources.
} 
a combined search to cover the whole island without each player perfectly duplicating the locations searched by its rival; this ensures that for a sufficiently small island, there is a positive-payoff strategy for both players.

This has potential policy implications. A government agency or regulator can improve welfare by insisting on some publicly available information or announcement by innovating firms (this could be a condition of receiving government subsidies or approval). Indeed, the amount of information contained in these statements need not be onerous to help the players avoid the extreme dissipation of value. This provides another justification for public announcements regarding intent to file patent applications and for the requirement of pharmaceutical companies to register with the industry regulator information regarding pending clinic trials.

\subsection{Perfect coordination $(\alpha=1)$}

We next consider perfectly coordinated search. ${ }^{21}$ There are many situations when it is possible to avoid duplication. If, for example, players can uniquely order all potential projects in a line, the two players have a choice to start searching from the left or the right, avoiding duplication. (This game effectively has two asymmetric equilibria, with each player commencing their search from different ends.) Another example is when the treasure is located on one of two separate islands; again, the players can avoid duplication by searching the island not occupied by their rival. Similar intuition applies when search requires some machinery (digging equipment, for instance). Not only does this provide observability as to the location of a rival's search, it might also make duplication physically impossible. $^{22}$ Perfect coordination is also consistent with one of the firms being able to adjust their search plans in the second stage of each period, as discussed at the beginning of Section 3.2.

When $\alpha=1$ equation (1) transforms to

$$
J=\min \left\{x, I_{1}+I_{2}\right\}
$$

Based on Lemma 3, define $d$ to be the maximum number of search periods when $\alpha=1$. One then can construct the sequence $\left\{\Psi_{s}\right\}_{s=1}^{d}$ and find all SMPE. This is called the valueiteration procedure, which is equivalent to using backward induction. In general, there will be multiple SMPE. As discussed earlier, in the case of multiplicity we focus on the SSMPE.

\footnotetext{
${ }^{21}$ An equivalent perfect coordination assumption is made in the previous R\&D literature; see for example, Cardon and Sasaki (1998) and Konrad (2014).

${ }^{22}$ Moreover, a third party like a government can often help firms avoid duplication. For example, patents prevent rivals encroaching on a firm's established area of expertise. Similarly, governments issue exclusive exploration licences to mining companies.
} 
We first define the polynomials $P_{s}(x)$ and critical island sizes $\chi_{s}$, for $s=1, \ldots, d,{ }^{23}$

$$
\begin{gathered}
P_{s}(x)=-\frac{1}{2}(1-x)^{2}+\frac{1}{2^{s}}(1-x)+\frac{(4 \delta)^{s}-4 \delta}{2(4 \delta-1) 4^{s}} \text { for } s=1, \ldots, d \\
\chi_{s}=1-\frac{3 \delta+(4 \delta)^{s}(\delta-1)}{2^{s}(4 \delta-1)} \text { for } s=1, \ldots, d-1 \text { and } \\
\chi_{d}=1+\frac{1}{2^{d}}\left(\sqrt{\frac{1-(4 \delta)^{d}}{1-4 \delta}}-1\right) \text { where } d=\left\lceil\frac{\ln \left(\left(\delta^{2}+2 \delta\right) /(1-\delta)^{2}\right)}{\ln (4 \delta)}\right\rceil .
\end{gathered}
$$

Next we give a complete characterization of the SSMPE when $\alpha=1$ in the following proposition.

Proposition 3. If $\alpha=1$, the non-trivial SSMPE exists if and only if $x \leq \chi_{d}$ and is described as follows. The search intensity of each firm is

$$
I(x)=\left\{\begin{array}{lrr}
\left.\frac{x}{2}, \delta\right)(1-x)+\delta / 2 \\
\frac{(1-\delta}{2 \delta}, & \text { if } & \chi_{1}<x \leq \chi_{1}, \\
\vdots & & \\
\frac{(1-\delta)(1-x)+\delta / 2^{s-1}}{2 \delta}, & \text { if } & \chi_{s-1}<x \leq \chi_{s} \\
\vdots & & \\
\frac{(1-\delta)(1-x)+\delta / 2^{d-1}}{2 \delta}, & \text { if } & \chi_{d-1}<x \leq \chi_{d}
\end{array}\right.
$$

and the value function for each firm is

$$
V(x)=\Psi(x) / x=\left\{\begin{array}{llr}
P_{1}(x) / x, & \text { if } & x \leq \chi_{1}, \\
\vdots & & \\
P_{d}(x) / x, & \text { if } & \chi_{d-1}<x \leq \chi_{d} .
\end{array}\right.
$$

Proof. See Appendix C.

Proposition 3 describes a finite horizon search for sufficiently small islands. In the nontrivial SSMPE duopolists plan to search the island for at most $d$ periods when $x \leq \chi_{d}$. There are $d$ possibilities, depending on the island size. If $x \leq \chi_{1}$, the duopolists search the island in just one period. If $x \in\left(\chi_{1}, \chi_{2}\right]$, the firms plan to search the island for at most two periods. In general, the duopolists plan to search the island for at most $s$ periods for values of $x \in\left(\chi_{s-1}, \chi_{s}\right]$, where $s=2, \ldots, d$.

As one can see from Proposition 3, the equilibrium search intensity $I(x)$ is a spline of degree one on the interval $\left[0, \chi_{d}\right]$ with knots $\chi_{1}, \ldots, \chi_{d} \cdot{ }^{24}$ Let us characterize the equilibrium

\footnotetext{
${ }^{23}$ Some of the expressions below are presented as ratios for expositional purposes. In particular, note that both numerators and denominators in $P_{s}, \chi_{s}, \chi_{d}$ and $d$ are equal to zero when $\delta=1 / 4$. These expressions are then defined by their limits as $\delta \rightarrow 1 / 4$.

${ }^{24} \mathrm{~A}$ spline is a special function defined piecewise by polynomials, see for example Ahlberg, Nielson, and Walsh (1967).
} 

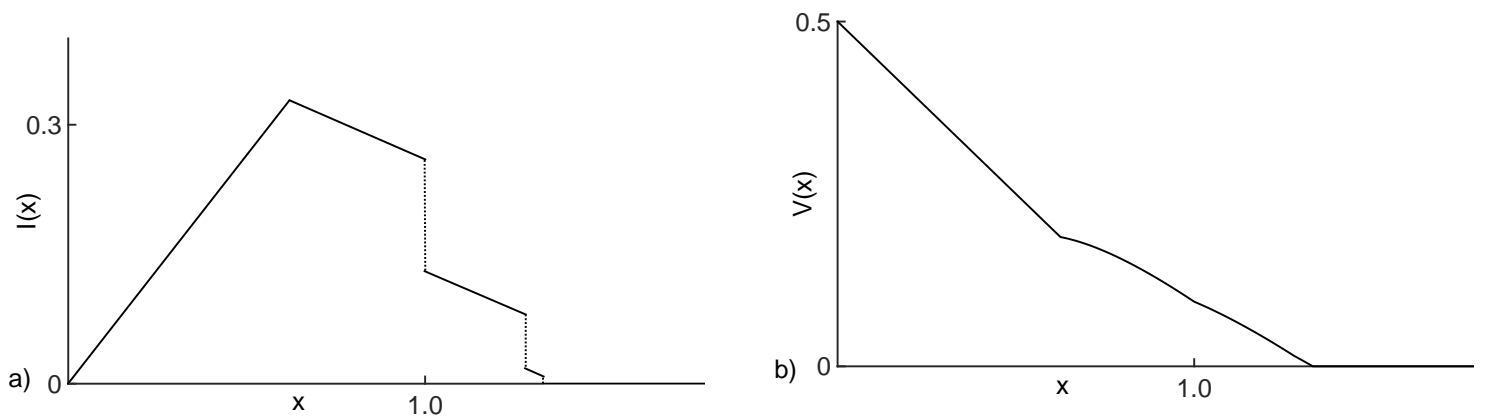

Figure 1: The equilibrium search intensity and value for duopolists when $\delta=0.75$.

search intensity in the following corollary.

Corollary 1. If $\alpha=1$ and $x \leq \chi_{d}$, the equilibrium search intensity $I(x)$ is a piece-wise linear, discontinuous and quasiconcave function.

Proof. See Appendix C.

Figure 1a illustrates the equilibrium search intensity for different island sizes in the SSMPE for each duopolist when $\delta=0.75$. In this case $d=4$. The equilibrium search intensity is a discontinuous function. At each knot of the spline $\chi_{s}$, the equilibrium strategy of a firm switches from searching for $s$ periods to searching for $s+1$ periods. The fact that each firm maximizes its own value, not total surplus, leads to this discontinuity. The discontinuities allow one to discern the intertemporal path of search by reading from right to left. Observe that, conditional on the search being conducted in 4 periods, the equilibrium search intensity is increasing in the first 3 periods. In the last period the equilibrium search intensity decreases. This is due to the fact that, while in the last period the duopolists search all of the remaining land, this search area in the last period can in fact be smaller than the area searched in the second-to-last period.

The following corollary characterizes the value function $V(x)$, as derived in Proposition 3.

Corollary 2. If $\alpha=1$ and $x \leq \chi_{d}$, the value function $V(x)$ is a continuous and monotonically decreasing function.

Proof. See Appendix C.

Figure $1 \mathrm{~b}$ illustrates the value function when $\delta=0.75$. Observe that the value function is monotonically decreasing with the size of the unsearched area because it is harder to find the treasure on a larger island. However, the value function is not smooth; the slope of the value function changes at knots $\chi_{s}$, coinciding with the discontinuities in the equilibrium search intensity. 


\subsection{The possibility of futile search when $\alpha=1$}

So far we have assumed that the treasure is located on the island with certainty. To add some extra realism, here we analyze the possibility of futile search. Specifically, we consider that the probability that the treasure exists on an island of size $x^{\prime}$ be $p \in(0,1]$ for the case when $\alpha=1$.

One possible interpretation of futile search is that the treasure is located on the island for sure but players may search only some part of the island. Extend the island to a size $x=x^{\prime} / p$ with the convention that the treasure belongs to the island of size $x$ with certainty, but only the portion $x^{\prime}$ can be searched, while the remainder is not accessible. That is, we assume the treasure is located with uniform probability on the area of size $x$. Further, we denote the portion of the island inaccessible by the players as $\gamma=x-x^{\prime}$.

Using this new structure, equation (4) transforms to

$$
V(x)=\max _{I_{i} \in[0, x-\gamma]}\left\{-I_{i}+\frac{J-I_{j}}{x}+\delta\left(1-\frac{J}{x}\right) V(x-J)\right\},
$$

where

$$
J=\min \left\{x-\gamma, I_{1}+I_{2}\right\}
$$

The only difference from the main model is that an area $\gamma \geq 0$ cannot be searched. As before, it is convenient to introduce the function $\Psi(x) \equiv x V(x)$. Equation (12) transforms to

$$
\Psi(x)=\max _{I_{i} \in[0, x-\gamma]}\left\{J-I_{j}-x I_{i}+\delta \Psi(x-J)\right\}
$$

Next we show that the problem with $\gamma>0$ can be transformed to the original problem with $\gamma=0$.

Proposition 4. Substitutions $x^{\prime}=\frac{x-\gamma}{1-\gamma}$ and $\Psi^{\prime}=\frac{\Psi}{(1-\gamma)^{2}}$ transform problem (14) into problem (7).

Proof. See Appendix C.

Proposition 4 implies that the problem (14) can be derived from (7) by a linear transformation. Intuitively, both (7) and (14) represent a multi-player cake eating problem. In this game, each player's strategy depends only on the remaining cake size (be it $x$ or $x-\gamma$ ), making both problems isomorphic. Therefore the perturbed model retains the properties of the original. In particular, searching becomes more attractive over time and, as a result, in any non-trivial equilibrium, players will continue to search until they either find the treasure or have searched the entire accessible region.

Figure 2 illustrates the equilibrium search intensity $I(x)$ (panel a)) and the value function $V(x)$ (panel b)) when $\delta=0.75$ and $\gamma=0.5$. The substitution $x^{\prime}=2 x-1$ transforms Figure 2 a to Figure 1a. Note that for $x \leq \gamma$, search is not possible. When $x>\gamma$, because of the transformation, search intensity appears more sensitive to island size.

On the other hand, in Figure $2 \mathrm{~b}$ the value function is very different from the one represented in Figure 1b. The transformation from $\Psi$ back to the original value function 

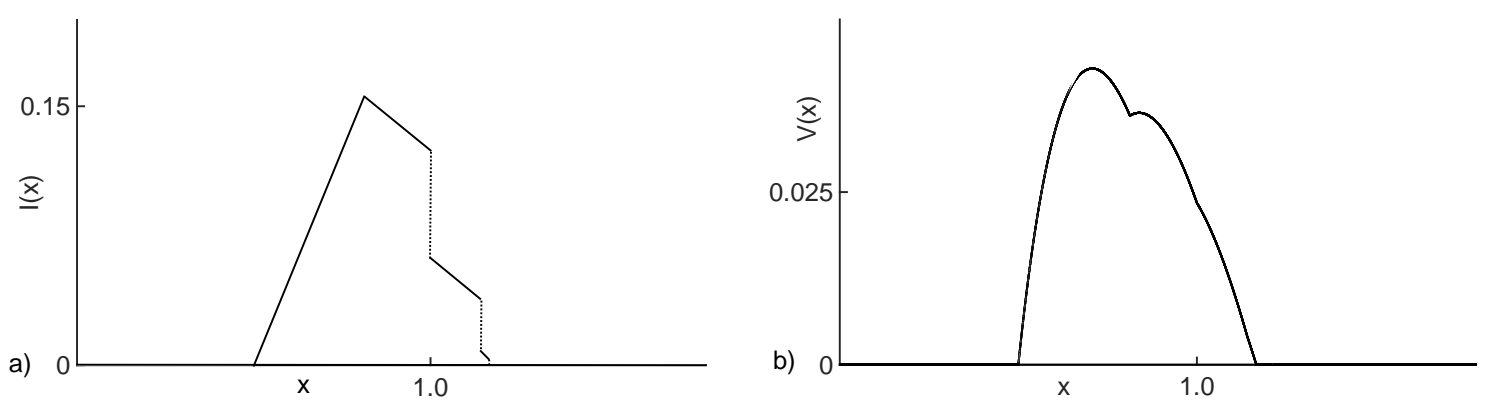

Figure 2: $I(x), V(x)$ when $\delta=0.75, \gamma=0.5$.

creates an additional effect. When $x \approx \gamma$, an increase in $x$ leads to a significant increase in the probability that the treasure is located on the part of the island that can be searched. This leads to a sharp increase in value, as shown in Figure 2b. However, once the island is significantly larger than $\gamma$, further increases in $x$ have a relatively small impact on the probability that the treasure belongs to the searchable part of the island. In this case, the increase in the cost of searching the entire island has a larger effect, and value declines in $x$. Consequently, there is a non-monotonic relationship between the value and island size. As in the main model, and for the same reasons, the value function is not smooth.

\subsection{Coordination and dynamics}

Let us now compare investment dynamics with independent and perfectly coordinated search. Figure 3 illustrates the equilibrium search intensity of each player, $I(x)$ (panel a)), and the combined search, $J(x)$ (panel b)), when $\delta=0.75$, both when $\alpha=0$ and $\alpha=1$. For small island sizes, the players search the entire island in a single period under perfect coordination. This leads to a linear relationship between $x$ and both $I(x)$ and $J(x)$ for small $x$. Now consider uncoordinated search when $x$ is small. Because of duplication, each firm searches more than they would with coordination. However, jointly the area searched by the two rivals is actually less with independent search, as their combined search does not cover the whole island (Lemma 2). With larger island sizes, search intensity in any one period is complicated by the sequencing of search over time, and it is possible for both individual and combined search to proceed more quickly without coordination. ${ }^{25}$ Furthermore, as noted above, the value from search with duplication is always zero, whereas with coordination there is a positive value of search for each player provided $x<\chi_{d}$.

\section{Discussion}

This paper considers duplication of search in a model with two players seeking to discover a prize of known value that is hidden in an unknown location. We model search as a

\footnotetext{
${ }^{25}$ Specifically, when $\delta \rightarrow 1, \chi_{2} \rightarrow 3 / 4$. In the neighborhood to the right of $\chi_{2}$ when $\delta \approx 1$, both individual and combined search intensities are higher with independent search.
} 

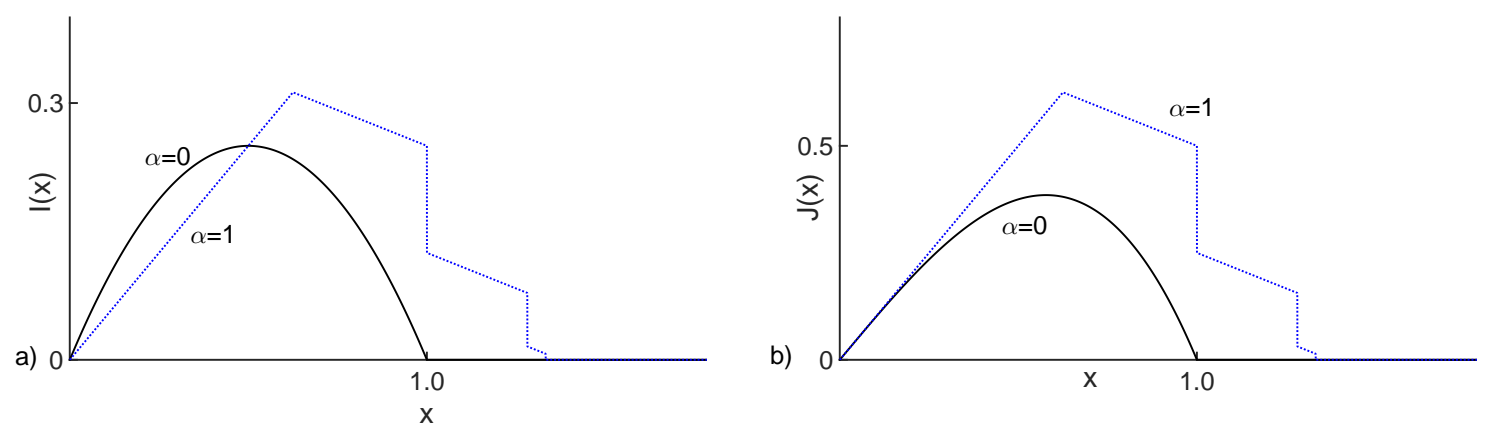

Figure 3: $I(x), J(x): \alpha=0$ is concave, $\alpha=1$ is piecewise linear; $\delta=0.75$.

dynamic R\&D race in a non-stationary environment and a finite search space. We show that duplication can be extreme - when the players are unable to coordinate their search at all, the unique equilibrium involves complete rent dissipation. Coordination, be it partial or perfect, can help alleviate some of these duplication issues, allowing players to earn positive expected returns. Consequently, this model has a clear implication for the role of a third party (a government, industry group or regulator) in facilitating coordination in research environments.

Several caveats relating to the model are worth commenting on. First, we use the SMPE as our equilibrium solution concept. There also exist asymmetric Markov perfect equilibria, where in every period each player searches a different amount. Using a similar approach, we consider an example of an asymmetric Markov perfect equilibrium in Appendix C. Second, there exist other non-Markovian symmetric equilibria that rely on trigger strategies similar to those in Compte and Jehiel (2004). In the case of small islands, the only symmetric equilibrium that exists is the SMPE. Players can always finish the search in one period, so trigger strategies are not useful in supporting cooperation. In contrast, in the case of large islands with a large number of potential search periods, trigger strategies may improve payoffs and additional equilibria can exist. Third, in the model we assume linear search costs. In a complementary paper, Matros and Smirnov (2011) focus on the comparative statics of monopoly versus duopoly and multi-player games allowing for quadratic costs.

Finally, in this paper we derive the search intensity and value generated for the players when there is no coordination $(\alpha=0)$ and when there is perfect coordination of search $(\alpha=1)$. We also derive the search intensity and value generated when there is partial coordination $(\alpha \in(0,1])$ and search is completed in one period. We have not solved the remaining case in which there is partial coordination when it is optimal to complete search of the island in more than one period. Deriving a complete analytical solution for the partial coordination case is beyond the scope of this project and we leave it for future research. 


\section{Appendix A}

For notational simplicity, we omit time subindexes. Here we provide micro-foundations as to why the area of duplication with independent search is $\frac{I_{1} I_{2}}{x}$ (see footnote 11).

Without loss of generality, let us model the unsearched area at a given point in time by considering a segment $[0, x]$. We assume that $[0, x]$ can be partitioned into $N$ equally-sized segments, where $N$ is large. There are $\frac{N I_{1}}{x}$ and $\frac{N I_{2}}{x}$ segments that are searched by players 1 and 2 respectively. We consider the limit when $N$ goes to infinity, and therefore we can ignore issues of discreteness.

We use the search of player 1, without loss of generality, as a reference by reordering all the segments so that the segments searched by player 1 are closest to the left corner of the original $[0, x]$. As a result, $[0, x]$ is divided into two parts: $\left[0, I_{1}\right]$ where player 1 searches and $\left[I_{1}, x\right]$ where player 1 does not search. Given search is independent, $\frac{N I_{2}}{x}$ segments are randomly selected among all $N$ segments. As a consequence, search duplication is given by the amount of search the second player undertakes in the $\left[0, I_{1}\right]$ segment.

Let us define $k$ as the number of small segments that land in the $\left[0, I_{1}\right]$ segment, describing the resulting number of successes. There are $n=\frac{N I_{2}}{x}$ draws, without replacement, from a population of size $N$ that contain $K=\frac{N I_{1}}{x}$ successes, wherein each draw is either a success or a failure. $k$ is a random variable with a hypergeometric distribution. As the size of each small segment is $\frac{x}{N}$, the area of duplicated search is equal to $A=\frac{k x}{N}$. Consequently, search duplication is distributed with the mean of $E[A]=\frac{I_{1} I_{2}}{x}$ and the variance of $\operatorname{Var}[A]=\frac{I_{1} I_{2}\left(1-I_{1} / x\right)\left(1-I_{2} / x\right)}{N-1}$. As $N \rightarrow \infty$, the distribution of $A$ becomes degenerate with the probability mass function equal to 1 at $\frac{I_{1} I_{2}}{x}$.

\section{Appendix B}

In this Appendix we modify the basic model to allow a non-uniform distribution for the location of the treasure. We investigate the cases of independent search $(\alpha=0)$ and perfect coordination $(\alpha=1)$ separately.

Consider $n \geq 2$ islands, indexed by $k=1, \ldots, n$. Assume that within each island there is an equal probability that the treasure will be located at any given point. Each island $k$ is described by its size $x^{(k)}$ and the probability $p^{(k)}$ that the treasure is located on the island. The attractiveness of island $k$ for search is determined by the probability of finding the treasure per unit area of the island. Without loss of generality, we order islands according to this measure:

$$
\frac{p^{(1)}}{x^{(1)}}>\frac{p^{(2)}}{x^{(2)}}>\cdots>\frac{p^{(n)}}{x^{(n)}}>0 \text { with } p=\sum_{k=1}^{n} p^{(k)} \leq 1,
$$

where $p$ is the probability the treasure is located on one of the islands. By adjusting the vector of island sizes and probabilities, we can approximate arbitrary distributions over the location of the treasure. ${ }^{26}$

Each player $i$ now has to choose how to structure his search across the $n$ islands. We denote the current state by the vectors $\boldsymbol{X}=\left(x^{(1)}, \ldots, x^{(n)}\right)$ and $\boldsymbol{p}=\left(p^{(1)}, \ldots, p^{(n)}\right)$ and the current value to player $i$ as $V(\boldsymbol{X}, \boldsymbol{p})$. Player $i$ chooses the policy functions $I_{i}^{(k)}(\boldsymbol{X}, \boldsymbol{p})$, for $k=1, \ldots, n$, which

\footnotetext{
${ }^{26}$ We follow recent papers of Erat and Krishnan (2012) and Konrad (2014) in modelling non-uniform distributions as we partition the landscape into sites (islands) described by different probabilities.
} 
describe the extent of simultaneous search he undertakes on each of the $n$ islands in the current period. As in the main model, if the treasure is not found in the current period, the unsearched area of each island $k$ shrinks to

$$
x_{t+1}^{(k)}=x_{t}^{(k)}-J_{t}^{(k)},
$$

where $J_{t}^{(k)}=\min \left\{x_{t}^{(k)}, I_{1, t}^{(k)}+I_{2, t}^{(k)}-(1-\alpha) I_{1, t}^{(k)} I_{2, t}^{(k)} / x_{t}^{(k)}\right\}$ and $\boldsymbol{J}=\left(J^{(1)}, \ldots, J^{(n)}\right)$. Following the current period search, the probability of finding the treasure in each island $k$ updates according to Bayes' rule as follows:

$$
p_{t+1}^{(k)}=\frac{p_{t}^{(k)}\left(1-J_{t}^{(k)} / x_{t}^{(k)}\right)}{1-\sum_{m=1}^{n} p_{t}^{(m)} J_{t}^{(m)} / x_{t}^{(m)}} .
$$

The Bellman equation faced by each player $i$ is then

$$
\begin{aligned}
& V(\boldsymbol{X}, \boldsymbol{p})=\max _{\left\{I_{i}^{(k)} \in\left[0, x^{(k)}\right]\right\}_{k=1}^{n}}\left\{\sum_{k=1}^{n}\left(-I_{i}^{(k)}+\frac{p^{(k)}\left(J^{(k)}-I_{j}^{(k)}\right)}{x^{(k)}}\right)\right. \\
&\left.+\delta\left(1-\sum_{k=1}^{n} \frac{p^{(k)} J^{(k)}}{x^{(k)}}\right) V\left(\boldsymbol{X}^{\prime}, \boldsymbol{p}^{\prime}\right)\right\},
\end{aligned}
$$

where transitions $\boldsymbol{X} \rightarrow \boldsymbol{X}^{\prime}$ and $\boldsymbol{p} \rightarrow \boldsymbol{p}^{\prime}$ are specified by relationships (16) and (17). The first term describes the current period costs and expected benefit of search across all $n$ islands. The second term represents the continuation value of the remaining vector of unsearched islands. In a similar manner as to how we defined the operator $B$ in equation (8) from the Bellman equation (7), here we introduce operator $\bar{B}$ based on equation (18).

\section{Independent search $(\alpha=0)$}

We first reexamine the case of independent search.

Proposition 5. If $\alpha=0$, in the unique SMPE, $I^{(k)}(\boldsymbol{X}, \boldsymbol{p})=\max \left\{0, x^{(k)}-\left(x^{(k)}\right)^{2} / p^{(k)}\right\}$ where $k \in\{1, n\}$ and $V(\boldsymbol{X}, \boldsymbol{p}) \equiv 0$.

Proof. See Appendix C.

Proposition 5 suggests that the extreme dissipation result of the basic model extends to heterogeneous distributions over the location of the treasure. That is, for any distribution, when $\alpha=0$ duplication is so egregious that the entire value of the treasure is squandered in equilibrium. The pattern of search has the following characteristics. First, in each period only islands that have $p_{t}^{(k)}>x_{t}^{(k)}$ will be searched in equilibrium, and all of these islands will be searched simultaneously by both players, with the intensity of search in island $k$ increasing in the attractiveness of search, given by equation (15). Second, after search is completed in any period, $p_{t}^{(k)}$ updates to $p_{t+1}^{(k)}$ according to Bayes' rule (17). As a result, search could expand to include new islands. This occurs for islands $k$ with $p_{t}^{(k)} \leq x_{t}^{(k)}$ and $p_{t+1}^{(k)}>x_{t+1}^{(k)}$. Finally, note that in a similar manner to the uniform distribution case, in equilibrium none of the islands will ever be completely searched.

\section{Perfect coordination $(\alpha=1)$}

Now consider perfect coordination. 
Proposition 6. If $\alpha=1$,

(1) in any SMPE, island $k$ is searched in period $t$ only if islands $1, \ldots, k-1$ are exhausted in period $\tau$, where $\tau \leq t$;

(2) there exists finite $T$ such that

(a) any non-trivial SMPE involves $J_{t}^{(k)}>0$ for $0 \leq t \leq T$ for at least one $k$, and $x_{T+1}^{(k)}=0$ for all $k$; and

(b) all SMPE can be obtained in T steps. Specifically,

$$
V_{0} \equiv 0, \quad V_{s} \equiv \bar{B} V_{s-1} \quad \text { for } \quad s=1,2, \ldots, T, \quad \text { and } \quad V=V_{T} .
$$

Proof. See Appendix C.

This proposition outlines the outcome when there are multiple islands that differ. In this case, part (1) states that the players will search the islands in order of their attractiveness, undertaking an exhaustive search before moving onto the next most attractive island. This is a similar result to Choi and Gerlach (2014) in which researchers move from the most attractive option down. This is a significantly different search pattern from one we observed in the independent search case studied earlier in this section. Part (2) extends the result in Lemma 3 with partial coordination, showing that search will be successful in finite time when there is perfect coordination and multiple islands. This ensures a positive expected return provided the project is close to completion. Taken together, Propositions 5 and 6 demonstrate the key role that coordination has in facilitating that all rents are not completely dissipated in the case of a non-uniform distribution regarding the location of the treasure.

\section{Appendix $\mathrm{C}$}

For notational simplicity, when convenient we omit firm and time subindexes.

\section{Proof of Lemma 1}

We introduce the new variables $\tilde{x}=c x / R, \tilde{I}_{i}=c I_{i} / R, \tilde{I}_{j}=c I_{j} / R$ and $\tilde{J}=c J / R$ and substitute them into problem (2). This substitution results in

$$
\bar{V}(\tilde{x}, 1, R)=\max _{\tilde{I}_{i} \in[0, \tilde{x}]}\left\{-\tilde{I}_{i} R+\frac{\tilde{J}-\tilde{I}_{j}}{\tilde{x}} R+\delta\left(1-\frac{\tilde{J}}{\tilde{x}}\right) \bar{V}(\tilde{x}-\tilde{J}, 1, R)\right\},
$$

where $\bar{V}(\tilde{x}, 1, R)=V(x, c, R)$. Next, substitute $\overline{\bar{V}}(\tilde{x}, 1,1)=\bar{V}(\tilde{x}, 1, R) / R$ into equation (19) to derive

$$
\overline{\bar{V}}(\tilde{x}, 1,1)=\max _{\tilde{I}_{i} \in[0, \tilde{x}]}\left\{-\tilde{I}_{i}+\frac{\tilde{J}-\tilde{I}_{j}}{\tilde{x}}+\delta\left(1-\frac{\tilde{J}}{\tilde{x}}\right) \overline{\bar{V}}(\tilde{x}-\tilde{J}, 1,1)\right\},
$$

which coincides with problem (2) when $R=c=1$. Consequently, $V(x, c, R) / R=V(c x / R, 1,1)$ proving the Lemma. 


\section{Proof of Lemma 2}

Substitute $I_{1}=x-\varepsilon_{1}, I_{2}=x-\varepsilon_{2}$ and $\alpha=0$ into (1) to derive

$$
J=x-\varepsilon_{1} \varepsilon_{2} / x .
$$

Part i) follows directly.

To prove part ii) assume that $I_{1}=I_{2}$. Condition $J \geq x$ transforms to $I_{i} \geq \frac{x}{1+\sqrt{\alpha}}$. This concludes the proof.

\section{Proof of Proposition 1}

In the proof below we follow the argument presented in the proof of Proposition 1 in Ericson and Pakes (1995). ${ }^{27}$ Introduce the following operator $\tilde{B}$ defined by

$$
(\tilde{B} V)(x) \equiv \max _{I \in[0, x]}\left\{-I+\frac{J-I_{j}}{x}+\delta\left(1-\frac{J}{x}\right) V(x-J)\right\},
$$

where the solution has to satisfy $I=I_{j}$ and $J=I+I_{j}-I I_{j} / x$. By the contraction property of the operator defined in equation $(20), V(x)=\lim _{s \rightarrow \infty} V_{s}(x)$, where $V_{s}(x) \equiv \tilde{B} V_{s-1}(x) \equiv \tilde{B}^{s} V_{0}$ and $V_{0} \equiv 0$. The remainder of the proof follows by induction. $V_{0} \equiv 0$, so an induction argument for $s=0$ is satisfied. Assume that $V_{s} \equiv 0$, and let us show that $V_{s+1} \equiv 0$. Substituting $V_{s} \equiv 0$ into the right hand side of equation (20), cancels out the continuation term on the right, yielding

$$
V_{s+1}(x)=\tilde{B} V_{s}(x) \equiv \max _{I \in[0, x]}\left\{-I+\frac{I}{x}\left(1-\frac{I_{j}}{x}\right)\right\} .
$$

First, consider when $x \leq 1$. If the search by the other player $I_{j}<x-x^{2}$, it is a dominant strategy for player $i$ to choose $I(x)=x$. If, on the other hand, search by the other player $I_{j}>x-x^{2}$, it is a dominant strategy for player $i$ to opt for $I(x)=0$. Moreover, if search by the other player $I_{j}=x-x^{2}$, then player $i$ is indifferent among all $I \in[0, x]$. There is a unique symmetric equilibrium where both players search $I(x)=x-x^{2}$ and the value each receives is zero; that is, $V_{s+1} \equiv 0$. Taking the limit gives $V(x)=\lim _{s \rightarrow \infty} V_{s}(x) \equiv 0$.

Second, consider when $x>1$. Observe that the payoff in (21) is negative unless $I(x)=0$. This concludes the proof.

\section{Proof of Lemma 3}

Suppose (a) is not satisfied. First, if $I_{t}=0$ for some $t=\hat{t}$, the Markovian assumption implies search remains zero for $\forall t>\hat{t}$. Hence, there are finitely many search periods. Assuming that the game lasts an infinite number of periods, this means that search in some periods will take arbitrarily small amounts, i.e. for $\forall \varepsilon_{0}>0 \exists \varepsilon \in\left(0, \varepsilon_{0}\right)$ and $\exists t \in N$ such that $I_{t}=\varepsilon \ll x$. It also means that there will be an infinite number of such periods. From (7) it follows $\Psi(x)=$ $(1-x) \varepsilon+\delta \Psi(x-J)+o(\varepsilon)$, which can be rewritten as

$$
\Psi(x-J)=\frac{\Psi(x)-(1-x) \varepsilon+o(\varepsilon)}{\delta},
$$

\footnotetext{
${ }^{27}$ Also see Stokey, Lucas and Prescott (1989).
} 
where $J=2 \varepsilon+o(\varepsilon)$.

Let us prove that in this case $\Psi(x)$ must be arbitrarily close to zero. Assume on the contrary that $\Psi(x)$ is equal to some positive value that is not infinitely small. By iterating equation (22) a large number of times it is possible to show that $\exists \hat{x} \in(0, x)$ such that $\Psi(\hat{x})$ can be larger than any positive value. However, by construction $\Psi(\hat{x})=\hat{x} V(\hat{x}) \leq \hat{x}$. We have reached a contradiction, proving that $\Psi(x)$ must be arbitrarily close to zero.

For values of $x<1, \Psi(x)$ can not be arbitrary close to zero, because each player will have incentive to deviate by choosing $I=x-\varepsilon+O\left(\varepsilon^{2}\right)$ and generating $\Psi(x)=(1-x) x+O(\varepsilon)>0$. Note that this argument works even when $x$ is very small, because a given $x>0$ can not be infinitely small.

When $x=1$ a similar argument applies; in the first period a player has an incentive to deviate by making a positive contribution, for example $I=0.5$. In this case, $\Psi(1)=\delta \Psi(0.5-\varepsilon)+O(\varepsilon)$. Given that $\Psi(x)$ is not arbitrarily close to zero when $x<1, \Psi(1)$ is also not arbitrarily close to zero.

When $x>1$ a slightly different proof is used. For a given size of island $x_{1}>1$, each player makes a positive search $I_{1}>0$ in the first period. Assuming that the game lasts an infinite number of periods, search has to take arbitrarily small values, i.e. for $\forall \varepsilon_{0}>0 \exists \varepsilon \in\left(0, \varepsilon_{0}\right)$ and $\exists t \in N$ such that $I_{t}=\varepsilon$, where $x>1$ and $t>1$. It also means that there will be an infinite number of such periods. As shown above, in this case $\Psi(x)$ is arbitrarily small. However, in the first period players make a positive search $I_{1}>0$, which means they incur an immediate negative payoff of at least $\left(1-x_{1}\right) I_{1}$. Subsequently, they make additional searches which add additional negative payoffs. Overall, this argument implies that $\Psi\left(x_{1}\right)$ is negative, which is a contradiction.

Thus, $I_{t}$ cannot be arbitrarily close to zero, which means there exists $\zeta>0$ such that $I_{t}>\zeta \forall t$. Consequently in any non-trivial SMPE the project has to be finished in a finite number of periods. This establishes part (a) of the Lemma.

Given that the project has to be finished in a finite number of periods, backward induction can be applied. Namely, we assume that $\Psi_{0} \equiv 0$ and derive $\Psi_{1} \equiv B \Psi_{0}$. This allows us to find all potential SMPE of the game if players could search for at most one period. Then we derive $\Psi_{2} \equiv B \Psi_{1}$, which allows us to find all potential SMPE of the game if players could search for at most two periods. We continue this process until $T$ is reached and find all potential SMPE of the game if players could search for at most $T$ periods. This establishes part (b) of the Lemma and concludes the proof.

\section{Proof of Proposition 2}

As proved in Lemma 3, the finite application of operator $B$ represents the algorithm for solving the Bellman equation. Let us start from the end period of search process. What will be the value if players could only search for at most one period? Equation (7) transforms into

$$
\Psi_{1}(x)=\max _{I \in[0, x]}\left\{J-I_{j}-x I\right\} .
$$

When $x$ is small the benefit of search is higher than the cost. In the unique SMPE both players cumulatively search the whole island; that is, $J=I+I_{j}-(1-\alpha) I I_{j} / x=x$. Consequently, provided $x \leq \sqrt{\alpha}$, each player searches

$$
I=\frac{x}{1+\sqrt{\alpha}}
$$


Substituting (24) into (4) and (23) results in

$$
\Psi_{1}(x)=x V_{1}(x)=\frac{(\sqrt{\alpha}-x) x}{1+\sqrt{\alpha}} .
$$

Now we analyze the situation when players plan to search for at most two periods. Equation (7) in this case transforms into

$$
\Psi_{2}(x)=\max _{I \in[0, x]}\left\{J-I_{j}-x I+\delta \Psi_{1}(x-J)\right\} .
$$

The necessary condition for $I$ to be optimal in the interior of $[0, x]$, provided that search lasts for two periods, is

$$
\frac{\partial J}{\partial I}\left(1-\delta \frac{\sqrt{\alpha}-2(x-J)}{1+\sqrt{\alpha}}\right)-x=0
$$

Note that $\frac{\partial J}{\partial I}>0$ for $J \leq x$. This means when $x$ is small the left hand side is positive and the optimal solution is not interior. Consequently, in a two-period search path players search the whole island in one period. To find the critical value $\chi_{1}$ for which searching the whole island in one period stops being a dominant strategy, we use the condition that the corner solution $x=J$ coincides with the internal solution (26). From this we derive a unique solution

$$
\chi_{1}=\sqrt{\alpha}\left(1-\delta \frac{\sqrt{\alpha}}{1+\sqrt{\alpha}}\right) .
$$

This concludes the proof.

\section{Proof of Proposition 3}

\section{Construction of $\Psi_{1}$ and $V_{1}$}

Let us start from the end of the search process. What will be the value if players could only search for at most one period? Equation (7) transforms into

$$
\Psi_{1}(x)=\max _{I \in[0, x]}\left\{\min \left\{x, I+I_{j}\right\}-I_{j}-x I\right\} .
$$

It is evident that in the unique non-trivial SMPE the equilibrium $I$ can be described in the following way ${ }^{28}$ :

$$
I=x / 2, \text { if } x \leq 1 .
$$

If $x \leq 1$, then in the SMPE players search the whole island, $2 I=x$. Consequently, the solution of $(27)$ is

$$
\Psi_{1}(x)=P_{1}(x), \text { if } x \leq u_{1}=1
$$

where $u_{1}=1$ is the largest positive root of polynomial $P_{1}(x)=x(1-x) / 2$. For future reference note that

$$
P_{1}(x)=\frac{a_{1}}{2}(1-x)^{2}+\frac{b_{1}}{2}(1-x)+\frac{c_{1}}{2},
$$

\footnotetext{
${ }^{28}$ Note that if $x=1$, then any $I \in[0, x / 2]$ is optimal. We assume that players choose $I=x / 2$ in this case.
} 
where

$$
a_{1}=-1, \quad b_{1}=1, \quad c_{1}=0 .
$$

Define the value for each player (if the players can search the island for at most $k$ periods) as $V_{k}(x) \equiv \Psi_{k}(x) / x$, for any $x \geq 0$. From the above definition, it follows that

$$
V_{1}(x)=(1-x) / 2, \text { if } x \leq u_{1}=1 .
$$

\section{Construction of $\Psi_{2}$ and $V_{2}$}

What will be the value if players can search the island for at most two periods? In general there could be three possibilities, depending on the island size. The first possibility is that the players search the whole island in just one period. Intuitively this happens for small values of $x$ because it is too costly to wait for another period when the island is very small. The second possibility is that the players search the island for at most two periods. This happens for middle values of $x$. Finally, players can find search to be too costly. When the initial island is large (costs are very high), there is no non-trivial SMPE (mixed, asymmetric or trivial equilibria are the only possibilities).

We have already considered the first possibility in the previous section. Now we analyze the situation when players plan to search for at most two periods. The first step is to construct $\Psi_{2}(x)$. Equation (7) in this case transforms into

$$
\Psi_{2}(x)=\max _{I \in[0, x]}\left\{\min \left\{x, I+I_{j}\right\}-I_{j}-x I+\delta \Psi_{1}\left(\max \left\{0, x-I-I_{j}\right\}\right)\right\} .
$$

The necessary condition for $I$ to be optimal in the interior of $[0, x]$, provided that search lasts for two periods, is

$$
(1-x)-\delta \Psi_{1}^{\prime}(x-2 I)=0 .
$$

In order to continue the search for the second period, the remaining island size has to satisfy

$$
0 \leq x-2 I \leq u_{1}
$$

The sufficient condition for $I$ to be optimal in the interior of $[0, x]$ is satisfied because

$$
\Psi_{1}^{\prime \prime}(x-2 I)=a_{1}<0 .
$$

The way to proceed is to construct the equilibrium with the help of condition (32), and then show that the derived equilibrium satisfies condition (33).

From expressions (32) and (28), it follows that

$$
(1-x)-\delta\left(\frac{1-2(x-2 I)}{2}\right)=0 .
$$

Consequently,

$$
I=\frac{2(\delta-1) x+2-\delta}{4 \delta} .
$$

Substituting (34) into equation (31), we obtain a spline of degree two on the interval $\left[0, u_{2}\right]$ :

$$
\Psi_{2}(x)= \begin{cases}\Psi_{1}(x), & \text { if } \quad 0 \leq x \leq \chi_{1} \\ P_{2}(x), & \text { if } \quad \chi_{1}<x \leq u_{2}\end{cases}
$$


where $u_{2}>0$ is the largest positive root of polynomial

$$
P_{2}(x)=\frac{a_{2}}{2}(1-x)^{2}+\frac{b_{2}}{2}(1-x)+\frac{c_{2}}{2},
$$

with

$$
a_{2}=-1, \quad b_{2}=\frac{1}{2}, \quad \text { and } \quad c_{2}=\frac{\delta}{4} .
$$

In order to find $u_{2}$, we need to solve the quadratic equation $P_{2}\left(u_{2}\right)=0$. It is easy to check that

$$
u_{2}=1+\frac{\sqrt{4 \delta+1}-1}{4}
$$

The point $x=\chi_{1}$ is the first knot of the spline. When $x=\chi_{1}$ players are indifferent between searching the island for two periods or for just one period:

$$
\Psi_{1}\left(\chi_{1}\right)=\Psi_{2}\left(\chi_{1}\right) .
$$

From (29) and (36), we derive a unique solution

$$
\chi_{1}=1-\frac{\delta}{2} .
$$

Note that condition (33) is satisfied for any $x \in\left[\chi_{1}, u_{2}\right]$. Therefore, if the players can search the island for at most two periods, the SSMPE is a spline of degree one on the interval $\left[0, u_{2}\right]$ :

$$
I(x)=\left\{\begin{array}{llr}
\frac{x}{2}, & \text { if } & x \leq \chi_{1} \\
\frac{2(\delta-1) x+2-\delta}{4 \delta}, & \text { if } & \chi_{1}<x \leq u_{2}
\end{array}\right.
$$

and the value function is

$$
V_{2}(x)=\left\{\begin{array}{llr}
V_{1}(x), & \text { if } & x \leq \chi_{1} \\
P_{2}(x) / x, & \text { if } & \chi_{1}<x \leq u_{2}
\end{array}\right.
$$

We can describe the construction of $\Psi_{k}$ and $V_{k}$ now.

\section{Construction of $\Psi_{k}$ and $V_{k}$}

What will be the value if players can search the whole island for at most $k \geq 3$ periods? In general there could be $k+1$ possibilities, depending on the island size $x_{1}$. The players can plan to search the island for at most $1,2, \ldots, k$ periods in a non-trivial SMPE. It is also possible that there is no non-trivial SMPE.

Let us construct $\Psi_{k}(x)$. Equation (7) in this case transforms into

$$
\Psi_{k}(x)=\max _{I \in[0, x]}\left\{\min \left\{x, I+I_{j}\right\}-I_{j}-x I+\delta \Psi_{k-1}\left(\max \left\{0, x-I-I_{j}\right\}\right)\right\} .
$$

The necessary condition for $I$ to be the optimal value in the interior of $[0, x]$, provided that search lasts for $k$ periods, is

$$
(1-x)=\delta \Psi_{k-1}^{\prime}(x-2 I)
$$


In order to continue search for the next period, the new value of $x$ has to satisfy

$$
\chi_{k-2} \leq x-2 I \leq u_{k-1},
$$

where $\chi_{0}=0$. The sufficient condition for $I$ to be the optimal value in the interior of $[0, x]$ is satisfied if

$$
\Psi_{k-1}^{\prime \prime}(x-2 I)<0 .
$$

We will use condition (42) to find $I$, and then show that it satisfies conditions (43) and (44). Note that if function $\Psi_{k-1}(x)$ in (41) is a quadratic polynomial, then $\Psi_{k}(x)=B \Psi_{k-1}(x)$ has to be a quadratic polynomial as well. Since $P_{1}(x)$ and $P_{2}(x)$ are quadratic polynomials by $(29)$ and (36), any $P_{k}(x)$ can be represented in the following form:

$$
P_{k}(x)=\frac{a_{k}}{2}(1-x)^{2}+\frac{b_{k}}{2}(1-x)+\frac{c_{k}}{2}, \quad k \geq 1 .
$$

From condition (42) and expression (45), it follows that

$$
I=-\frac{(1-x)\left(1+\delta a_{k-1}\right)}{2 \delta a_{k-1}}-\frac{b_{k-1}}{4 a_{k-1}} .
$$

Define the largest root of polynomial $P_{k}(x)$ as $u_{k}$, and that value of $x$ such that players switch from planning to search the area for $k-1$ periods to planning to search the area for $k$ periods as knot $\chi_{k-1}$ :

$$
\Psi_{k-1}\left(\chi_{k-1}\right)=\Psi_{k}\left(\chi_{k-1}\right)
$$

For the moment, let us assume that equation (47) has a unique solution. The uniqueness of the solution will be proved below. Substituting (46) into equation (41), we obtain a spline of degree two on the interval $\left[0, u_{k}\right]$ :

$$
\Psi_{k}(x)= \begin{cases}\Psi_{k-1}(x), & \text { if } \quad 0 \leq x \leq \chi_{k-1} \\ P_{k}(x), & \text { if } \quad \chi_{k-1}<x \leq u_{k}\end{cases}
$$

where $P_{k}(x)$ is defined in (45). Therefore, if players plan to search the island for at most $k$ periods, then $I(x)$ is a spline of degree one on the interval $\left[0, u_{k}\right]$ :

$$
I(x)=\left\{\begin{array}{llr}
\frac{x}{2}, & \text { if } & x \leq \chi_{1}, \\
-\frac{(1-x)\left(1+\delta a_{1}\right)}{2 \delta a_{1}}-\frac{b_{1}}{4 a_{1}}, & \text { if } & \chi_{1}<x \leq \chi_{2}, \\
\vdots & & \\
-\frac{(1-x)\left(1+\delta a_{k-1}\right)}{2 \delta a_{k-1}}-\frac{b_{k-1}}{4 a_{k-1}}, & \text { if } & \chi_{k-1}<x \leq u_{k} ;
\end{array}\right.
$$

and the value function is

$$
V_{k}(x)= \begin{cases}V_{k-1}(x), & \text { if } \quad 0 \leq x \leq \chi_{k-1} \\ P_{k}(x) / x, & \text { if } \quad \chi_{k-1}<x \leq u_{k}\end{cases}
$$

Let us now find $a_{k}, b_{k}$, and $c_{k}$ for any $k \geq 2$. Using (41), (45) and (46), we get the following system of difference equations:

$$
a_{k}=-1, \quad b_{k}=-\frac{b_{k-1}}{2 a_{k-1}}, \quad c_{k}=\delta\left(c_{k-1}-\frac{b_{k-1}^{2}}{4 a_{k-1}}\right), \quad k \geq 2,
$$


where

$$
a_{1}=-1, \quad b_{1}=1, \quad c_{1}=0
$$

Derivation of $a_{k}, b_{k}$ and $c_{k}$

Let us now solve the system of difference equations (51). It is straightforward to derive

$$
a_{k}=-1 \quad \text { and } \quad b_{k}=\frac{1}{2^{k-1}} \text {. }
$$

Note that $a_{k} \neq 0$ and the system (51) is well defined.

The expression for $c_{k}$ in (51) can be simplified to

$$
c_{k}=\delta\left(c_{k-1}+1 / 4^{k-1}\right) .
$$

Introduce a new variable $e_{k}=c_{k} 4^{k}$. Equation (54) transforms to

$$
e_{k}=4 \delta\left(e_{k-1}+1\right)
$$

where $e_{1}=0$. The solution to this linear difference equation is $e_{k}=\frac{4 \delta-(4 \delta)^{k}}{1-4 \delta}$. Substitute $c_{k}=$ $e_{k} / 4^{k}$ to derive

$$
c_{k}=\frac{(4 \delta)^{k}-4 \delta}{(4 \delta-1) 4^{k}}
$$

\section{Derivation of $\chi_{k}$}

To find $\chi_{k}$, one needs to solve the quadratic equation $P_{k}\left(\chi_{k}\right)=P_{k+1}\left(\chi_{k}\right)$, namely

$$
a_{k}\left(1-\chi_{k}\right)^{2}+b_{k}\left(1-\chi_{k}\right)+c_{k}=a_{k+1}\left(1-\chi_{k}\right)^{2}+b_{k+1}\left(1-\chi_{k}\right)+c_{k+1}, \quad k \geq 1 .
$$

From equation (53), $a_{k}=a_{k+1}=-1$; consequently,

$$
\chi_{k}=1+\frac{c_{k+1}-c_{k}}{b_{k+1}-b_{k}} .
$$

Substitute $b_{k}$ and $c_{k}$ from equations (53) and (55) to derive the following indifference points

$$
\chi_{k}=1-\frac{3 \delta+(4 \delta)^{k}(\delta-1)}{2^{k}(4 \delta-1)} .
$$

Note that the solution to the quadratic equation $P_{k}\left(\chi_{k}\right)=P_{k+1}\left(\chi_{k}\right)$ is always unique.

\section{Derivation of $u_{k}$}

To find $u_{k}$, one needs to solve the quadratic equation $P_{k}\left(u_{k}\right)=0$, namely

$$
a_{k}\left(1-u_{k}\right)^{2}+b_{k}\left(1-u_{k}\right)+c_{k}=0, \quad k \geq 1 .
$$

Substituting $a_{k}=-1$ from equation (53) and solving the above quadratic equation gives

$$
u_{k}=1+\frac{\sqrt{b_{k}^{2}+4 c_{k}}-b_{k}}{2} .
$$


Note that with the help of (53) and (55), one can simplify:

$$
b_{k}^{2}+4 c_{k}=\frac{(4 \delta)^{k}-1}{4^{k-1}(4 \delta-1)} .
$$

Substitute equation (58) into equation (57) to get

$$
u_{k}=1+\frac{\sqrt{\frac{1-(4 \delta)^{k}}{1-4 \delta}}-1}{2^{k}} .
$$

Note that for the ease of exposition we present $\chi_{d}=u_{d}$ in the statement of the Proposition.

\section{Derivation of $d$}

One way to find the maximum number of search periods is to write the condition that the largest positive root of the quadratic polynomial $P_{k}(x)$ coincides with the largest positive root of the quadratic polynomial $P_{k+1}(x)$. This condition gives a critical value of $\delta$; for slightly larger values of $\delta$, there is an additional search period.

This means that $k$ is the smallest integer such that $\Psi_{k}(x) \equiv \Psi_{k+1}(x)$, or in other words $V(x) \equiv V_{k}(x)$. Specifically, when $\delta$ is at a critical value, $k$ has to be an integer and is the solution to the following equation:

$$
u_{k}(\delta, n)=t_{k}(\delta, n)
$$

Substitute $t_{d}$ and $u_{d}$ from equations (56) and (59) into equation (60) to get

$$
1+\frac{\sqrt{\frac{1-(4 \delta)^{d}}{1-4 \delta}}-1}{2^{d}}=1-\frac{3 \delta+(4 \delta)^{d}(\delta-1)}{2^{d}(4 \delta-1)} .
$$

Simplify the above expression to

$$
\sqrt{\frac{1-(4 \delta)^{d}}{1-4 \delta}}-1=-\frac{3 \delta+(4 \delta)^{d}(\delta-1)}{(4 \delta-1)} .
$$

Further simplifications give

$$
\sqrt{\frac{(4 \delta)^{d}-1}{4 \delta-1}}=\frac{\left((4 \delta)^{d}-1\right)(1-\delta)}{(4 \delta-1)}
$$

and

$$
\sqrt{\frac{(4 \delta)^{d}-1}{4 \delta-1}}(1-\delta)=1 .
$$

Square both sides of equation (61) to derive the maximum number of search periods: ${ }^{29}$

$$
d=\left\lceil\frac{\ln \left(\left(\delta^{2}+2 \delta\right) /(1-\delta)^{2}\right)}{\ln (4 \delta)}\right\rceil .
$$

\footnotetext{
${ }^{29}$ Note that when $\delta=1 / 4$ both numerator and denominator in $d$ contain $\ln (4 \delta)$ term, which means $d$ is not well defined. The interpretation of this expression for $d$ when $\delta=1 / 4$ is its limit as $\delta \rightarrow 1 / 4$.
} 


\section{Conditions (43) and (44) are satisfied}

Define that part of $x$ which player $i$ does not search in the current period by $y=x-I$ and the part of $x$ no player searches in the current period by $z=x-\left(I+I_{j}\right)=y-I_{j}$.

Next, show that condition (43) is satisfied; that is, $t_{k-2} \leq z \leq u_{k-1}$. Let us prove the first part $z \leq u_{k-1}$ by contradiction, assuming that $z>u_{k-1}$. Refer to equation (41), which is written below:

$$
\Psi_{k}(x)=\max _{I \in[0, x]}\left\{\min \left\{x, I+I_{j}\right\}-I_{j}-x I+\delta \Psi_{k-1}\left(\max \left\{0, x-I-I_{j}\right\}\right)\right\} .
$$

Given $x \geq z>u_{k-1} \geq \cdots \geq u_{2} \geq u_{1}=1$ and $I+I_{j} \leq x$, it follows that the first term $\min \left\{x, I+I_{j}\right\}-I_{j}-x I \leq(1-x) I$ has to be non-positive. If $z>u_{k-1}$, then the second term $\delta \Psi_{k-1}(z)$ is negative. That means the whole expression on the right of equation (41) has to be negative. Obviously that could not be an optimal choice for a player because by choosing $y=x$, that is, by not searching, a player can get the value of zero. Consequently, there is a contradiction, and condition $z \leq u_{k-1}$ is proved.

Now let us show that $\chi_{k-2} \leq z$. From equations (46), (53) and (55), it follows that $z(x)=$ $1-2^{1-k}-\frac{1-x}{\delta}$. It is easy to see that $z(x)$ is a monotonically increasing function in $x$. Consequently, it is sufficient to prove the above condition for $x=\chi_{k-1}$. Substituting values from (56) and simplifying gives

$$
\frac{3 \delta+(4 \delta)^{k-1}(\delta-1)}{\delta(4 \delta-1)} \leq \frac{1+2 \delta+2(4 \delta)^{k-2}(\delta-1)}{4 \delta-1} .
$$

Further simplifications result in $(4 \delta)^{k-1} \geq 4 \delta$, which is satisfied for any $k>2$.

Finally, let us show that condition (44) is satisfied; that is, $\Psi_{k-1}^{\prime \prime}(z)<0$. From equations (45), (48) and (53), it follows that $\Psi_{k-1}^{\prime \prime}(z)=a_{k-1}=-1$. This concludes the proof.

\section{Proof of Corollary 1}

First, $I(x)$ presented in Proposition 3 is a piece-wise linear function. Second, let us prove that $I(x)$ is not a continuous function. Given that $I(x)$ is piece-wise linear, discontinuities are only possible in the knots of the spline. Substitute $x=\chi_{s}$ into (49), when the project is planned to be finished in $s \geq 2$ periods, to derive

$$
I\left(\chi_{s}\right)=\frac{2-2 \delta+\delta / 2^{s-2}-2(1-\delta) \chi_{s}}{4 \delta} .
$$

Next, substitute $x=\chi_{s}$ into (49), when the project is planned to be finished in $s+1$ periods, to derive

$$
I\left(\chi_{s}\right)=\frac{2-2 \delta+\delta / 2^{s-1}-2(1-\delta) \chi_{s}}{4 \delta} .
$$

The difference between the expressions in (63) and (64) is $\Delta I\left(\chi_{s}\right)=1 / 2^{s+1}>0$.

Third, let us find the slope of $I(x)$. If the project is finished in one period then the slope is positive, $I^{\prime}(x)=\frac{1}{2}$, otherwise it is negative, $I^{\prime}(x)=\frac{\delta-1}{2 \delta}$. This proves quasiconcavity and concludes the proof. 


\section{Proof of Corollary 2}

First, $V(x)$ presented in Proposition 3 has to be continuous. Remember that knot $\chi_{s}$ is defined as an area size for which the duopolists are indifferent between planning to search the area for $s$ periods or for $s+1$ periods.

Second, to show that the value function is monotonically decreasing we need to show that $\forall s \geq 2$ the following inequality holds:

$$
V_{s}^{\prime}(x)=\left(\frac{P_{s}(x)}{x}\right)^{\prime}=a_{s}-\frac{a_{s}+b_{s}+c_{s}}{x^{2}}<0 .
$$

Substitution of $a_{s}=-1$ transforms (65) to

$$
b_{s}+c_{s}+x^{2}>1
$$

For a given $s$, the left hand side is increasing in $x$, which means it is sufficient to prove the inequality for $x=\chi_{s-1}$. Substitute $b_{s}, c_{s}$ and $\chi_{s-1}$ from (53),(54) and (56) to derive

$$
\frac{1}{2^{s-1}}+\left(\frac{(4 \delta)^{s-1}-1}{4^{s-1}(4 \delta-1)}\right) \delta+\left(1-\frac{3 \delta+(4 \delta)^{s-1}(\delta-1)}{2^{s-1}(4 \delta-1)}\right)^{2}>1
$$

Let us prove inequality (66) separately for $s \in[2,3]$ and for $s \in[4, d]$. When $s=2$, the inequality transforms to $2-3 \delta+\delta^{2}>0$, which is always satisfied for $\delta<1$. Similarly when $s=3$, the inequality transforms to $4+25 \delta-28 \delta^{2}+\left(3 \delta-4 \delta^{2}\right)^{2}>0$, which is also always satisfied for $\delta<1$.

From (61) when $d \geq 4$, it is the case that $\delta>1 / 2$. Simplify inequality (66) to

$$
\left(2^{s-1}-1\right)\left(2 \frac{(4 \delta)^{s-1}-1}{(4 \delta-1)}-1\right)(1-\delta)+\left(\frac{(4 \delta)^{s-1}-1}{(4 \delta-1)}+1-2^{s-1}\right) \delta+\left(\frac{\left((4 \delta)^{s-1}-1\right)(\delta-1)}{4 \delta-1}\right)^{2}>0
$$

The first and the third terms are positive for $s \geq 4$. The second term is increasing in $\delta$. Substitute $\delta=1 / 2$ to derive

$$
\left(\frac{(4 \delta)^{s-1}-1}{(4 \delta-1)}+1-2^{s-1}\right) \delta=\left(1+2+\cdots+2^{s-2}+1-2^{s-1}\right) \delta=0 .
$$

This proves the Corollary.

\section{Proof of Proposition 4}

Introduce $\tilde{x}=(x-\gamma) /(1-\gamma), \tilde{I}=I /(1-\gamma), \tilde{I}_{j}=I_{j} /(1-\gamma)$ and $\tilde{J}=J /(1-\gamma)$ and substitute them in problem (14). That results in

$$
\bar{\Psi}(\tilde{x})=\max _{\tilde{I} \in[0, \tilde{x}]}\left\{\min \left\{(1-\gamma)\left(\tilde{x}-\tilde{I}_{j}-x \tilde{I}\right),(1-\gamma)^{2}(1-\tilde{x}) \tilde{I}\right\}+\delta \bar{\Psi}(\tilde{x}-\tilde{J})\right\},
$$

where $\bar{\Psi}(\tilde{x})=\Psi(x)$. Substitute $\overline{\bar{\Psi}}(\tilde{x})=\bar{\Psi}(\tilde{x}) /(1-\gamma)^{2}$ and $\overline{\bar{\Psi}}(\tilde{x}-\tilde{J})=\bar{\Psi}(\tilde{x}-\tilde{J}) /(1-\gamma)^{2}$ into equation (67) to derive

$$
\overline{\bar{\Psi}}(\tilde{x})=\max _{\tilde{I} \in[0, \tilde{x}]}\left\{\tilde{J}-\tilde{I}_{j}-\tilde{x} \tilde{I}+\delta \overline{\bar{\Psi}}(\tilde{x}-\tilde{J})\right\}
$$


which coincide with problem (7). It follows that the substitutions $x^{\prime}=\frac{x-\gamma}{1-\gamma}$ and $\Psi^{\prime}=\frac{\Psi}{(1-\gamma)^{2}}$ allow to transform problem (14) into problem (7). This concludes the proof.

\section{Proof of Proposition 5}

Let us generalize the argument presented in Proposition 1 for multiple islands. Introduce the following operator $\bar{B}$ defined by

$$
\begin{aligned}
&(\bar{B} V)(\boldsymbol{X}, \boldsymbol{p})=\max _{\left\{I_{i}^{(k)} \in\left[0, x^{(k)}\right]\right\}_{k=1}^{n}}\left\{\sum_{k=1}^{n}\left(-I_{i}^{(k)}+\frac{p^{(k)}\left(J^{(k)}-I_{j}^{(k)}\right)}{x^{(k)}}\right)\right. \\
&\left.+\delta\left(1-\sum_{k=1}^{n} \frac{p^{(k)} J^{(k)}}{x^{(k)}}\right) V\left(\boldsymbol{X}^{\prime}, \boldsymbol{p}^{\prime}\right)\right\} .
\end{aligned}
$$

where transitions $\boldsymbol{X} \rightarrow \boldsymbol{X}^{\prime}$ and $\boldsymbol{p} \rightarrow \boldsymbol{p}^{\prime}$ are specified by (16) and (17) and the solution has to satisfy $I^{(k)}=I_{j}^{(k)}$ and $J^{(k)}=I^{(k)}+I_{j}^{(k)}-I^{(k)} I_{j}^{(k)} / x^{(k)}$ for $k=1, \ldots, n$. By the contraction property of the operator defined in equation (68), $V=\lim _{s \rightarrow \infty} V_{s}$, where $V_{s} \equiv \bar{B} V_{s-1} \equiv \bar{B}^{s} V_{0}$ and $V_{0} \equiv 0$. The remainder of the proof follows by induction. $V_{0} \equiv 0$, so an induction argument for $s=0$ is satisfied. Assume that $V_{s} \equiv 0$, and let us show that $V_{s+1} \equiv 0$. Substituting $V_{s} \equiv 0$ into the right hand side of equation (68), cancels out the continuation term on the right, yielding

$$
V_{s+1}\left(x^{(k)}\right)=\bar{B} V_{s}\left(x^{(k)}\right) \equiv \max _{\left\{I^{(k)} \in\left[0, x^{(k)}\right]\right\}_{k=1}^{n}}\left\{\sum_{k=1}^{n}\left(-I^{(k)}+\frac{p^{(k)} I^{(k)}}{x^{(k)}}\left(1-\frac{I_{j}^{(k)}}{x^{(k)}}\right)\right)\right\},
$$

where the solution has to satisfy $I^{(k)}=I_{j}^{(k)}$ for $k=1, \ldots, n$. The optimization problem (69) is separable in $k$, which means it is sufficient to solve each island's problem independently.

For each island $k$ consider separately when $p^{(k)} \geq x^{(k)}$ and when $p^{(k)}<x^{(k)}$. When $p^{(k)} \geq x^{(k)}$ if the search by the other player $I_{j}^{(k)}<x^{(k)}-\left(x^{(k)}\right)^{2} / p^{(k)}$, it is a dominant strategy for player $i$ to choose $I^{(k)}=x^{(k)}$. If, on the other hand, search by the other player $I_{j}^{(k)}>x^{(k)}-\left(x^{(k)}\right)^{2} / p^{(k)}$, it is a dominant strategy for player $i$ to opt for $I^{(k)}=0$. Moreover, if search by the other player $I_{j}^{(k)}=x^{(k)}-\left(x^{(k)}\right)^{2} / p^{(k)}$, then player $i$ is indifferent among all $I^{(k)} \in\left[0, x^{(k)}\right]$. There is a unique symmetric equilibrium where each player searches $I^{(k)}\left(x^{(k)}\right)=x^{(k)}-\left(x^{(k)}\right)^{2} / p^{(k)}$ and the value each receives is zero; that is, $V_{s+1} \equiv 0$. Taking the limit gives $V(x)=\lim _{s \rightarrow \infty} V_{s}(x) \equiv 0$.

Applying similar logics when $p^{(k)}<x^{(k)}$ gives the result that each player searches $I^{(k)}=0$. This proves the Proposition.

\section{Proof of Proposition 6}

Suppose (1) is not satisfied. This means that an island with a lower attractiveness is searched earlier than a more attractive island in equilibrium. If this were true, there would be a one-shot profitable deviation for either player, which is a contradiction.

Let us generalize the argument presented in Lemma 3 for multiple islands. Suppose (2a) is not satisfied. First, if $I_{t}^{(k)}=0 \forall k$ for some $t=\hat{t}$, the Markovian assumption implies search remains zero for $\forall t>\hat{t}$. Hence, there are finitely many search periods. Assuming that the game lasts an infinite number of periods, this means that search in some periods will involve arbitrarily small 
amounts, i.e. for $\forall \varepsilon_{0}>0 \exists \varepsilon \in\left(0, \varepsilon_{0}\right)$ and $\exists t \in N$ such that $\sum_{k=1}^{n} I_{t}^{(k)}=\varepsilon$. It also means that there will be an infinite number of such periods.

Without loss of generality assume that $\bar{n}$ islands are not wholly searched at $t$, and $\tilde{x}$ is the smallest unsearched area of such islands. Then $\varepsilon$ can be chosen such that $\varepsilon \ll \tilde{x}$, and from (18) it follows $V(\boldsymbol{X}, \boldsymbol{p})=\sum_{k=1}^{\bar{n}} \frac{I^{(k)}\left(p^{(k)}-x^{(k)}\right)}{x^{(k)}}+\delta\left(1-\sum_{m=1}^{\bar{n}} \frac{p^{(m)} J^{(m)}}{x^{(m)}}\right) V\left(\boldsymbol{X}^{\prime}, \boldsymbol{p}^{\prime}\right)$. This can be rewritten as

$$
V\left(\boldsymbol{X}^{\prime}, \boldsymbol{p}^{\prime}\right)=\frac{V(\boldsymbol{X}, \boldsymbol{p})-\sum_{k=1}^{\bar{n}} \frac{I^{(k)}\left(p^{(k)}-x^{(k)}\right)}{x^{(k)}}}{\delta\left(1-\sum_{m=1}^{\bar{n}} \frac{p^{(m)} J^{(m)}}{x^{(m)}}\right)}=\frac{V(\boldsymbol{X}, \boldsymbol{p})+O(\varepsilon)}{\delta} .
$$

Let us prove that in this case $V(\boldsymbol{X}, \boldsymbol{p})$ must be arbitrarily close to zero. Assume on the contrary that $V(\boldsymbol{X}, \boldsymbol{p})$ is equal to some positive value that is not infinitely small. By iterating equation (70) a large number of times it is possible to show that $\exists \hat{\boldsymbol{X}}=\left(\hat{x}^{(1)}, \ldots, \hat{x}^{(n)}\right)$ and $\hat{\boldsymbol{p}}=\left(\hat{p}^{(1)}, \ldots, \hat{p}^{(n)}\right)$ such that $\hat{x}^{(k)} \in\left(0, x^{(k)}\right) \forall k$ and $V(\hat{\boldsymbol{X}}, \hat{\boldsymbol{p}})$ can be larger than any positive value. However, by construction $V(\hat{\boldsymbol{X}}, \hat{\boldsymbol{p}}) \leq 1$. We have reached a contradiction, proving that $V(\boldsymbol{X}, \boldsymbol{p})$ must be arbitrarily close to zero.

For values of $\boldsymbol{X}$ and $\boldsymbol{p}$ where $p^{(k)}>x^{(k)}$ at least for some $k, V(\boldsymbol{X}, \boldsymbol{p})$ can not be arbitrary close to zero, because each player will have incentive to deviate by choosing $I^{(k)}=x^{(k)}-\varepsilon+O\left(\varepsilon^{2}\right)$ and generating $V(\boldsymbol{X})=p^{(k)}-x^{(k)}+O(\varepsilon)>0$.

For values of $\boldsymbol{X}$ and $\boldsymbol{p}$ where $p^{(k)}=x^{(k)}$ for some $k$ and $p^{(s)} \leq x^{(s)} \forall s \neq k$ a similar argument applies; in the first period a player has an incentive to deviate by making a positive contribution, for example he could deviate to $I^{(k)}=x^{(k)} / 2$. In this case, $V(\boldsymbol{X}, \boldsymbol{p}) \geq \delta V\left(\boldsymbol{X}^{\prime}, \boldsymbol{p}^{\prime}\right)+O(\varepsilon)$, where $\boldsymbol{X}^{\prime}$ satisfy the condition proved in the previous paragraph; namely, new values of $p^{(k)}$ and $x^{(k)}$ are such that $p^{(k)}>x^{(k)}$. Given that in the previous paragraph it is proved that $V\left(\boldsymbol{X}^{\prime}, \boldsymbol{p}^{\prime}\right)$ is not arbitrarily close to zero, $V(\boldsymbol{X}, \boldsymbol{p})$ is also not arbitrarily close to zero.

When $p^{(k)}<x^{(k)} \forall k$, a slightly different proof is used. For a given $\boldsymbol{X}$ and $\boldsymbol{p}$, each player makes a positive search $\Upsilon=\sum_{k=1}^{n} I^{(k)}>0$ in the first period. Assuming that the game lasts an infinite number of periods, search has to take arbitrarily small values, i.e. for $\forall \varepsilon_{0}>0 \exists \varepsilon \in\left(0, \varepsilon_{0}\right)$ and $\exists t \in N$, such that $\sum_{k=1}^{n} I_{t}^{(k)}=\varepsilon$. It also means that there will be an infinite number of such periods. As shown above, in this case $V(\boldsymbol{X}, \boldsymbol{p})$ is arbitrarily small. However, in the first period players make a positive search $\Upsilon$, which means they will incur an immediate negative payoff of at least $\Upsilon \max _{k}\left\{\frac{p^{(k)}-x^{(k)}}{x^{(k)}}\right\}$. Subsequently, they make additional searches, which add additional negative payoffs. Overall, this argument implies that $V(\boldsymbol{X}, \boldsymbol{p})$ is negative, which is a contradiction. Note that, if in the process, the condition $p^{(k)}<x^{(k)} \forall k$ ceases being satisfied, then $\exists k$ such that $p^{(k)} \geq x^{(k)}$; we have considered this case earlier.

Thus, $\sum_{k=1}^{n} I_{t}^{(k)}$ cannot be arbitrarily close to zero, which means there exists $\zeta>0$ such that $\sum_{k=1}^{n} I_{t}^{(k)}>\zeta \forall t$. Consequently in any non-trivial SMPE the project has to be finished in a finite number of periods. This establishes part (2a) of the Proposition.

The proof of part (2b) of the Proposition closely follows the proof of part (b) of Lemma 3. This concludes the proof.

\section{An example of asymmetric equilibria}

Assume that there is only one island and the players can perfectly coordinate $(\alpha=1)$. Consider the following two-period game. Player $i=1,2$ can search the island of size $x_{1}=0.9$ in the two potential periods, $t=1,2$, in order to find a treasure of value 1 . Payoffs are discounted at a common factor $\delta=0.75$. 
Let us solve this problem by backward induction. Consider the second period subgame in which the treasure was not found in the first period and the unsearched area of the island is $x \leq x_{1}$. Provided there is no duplication, each player has a dominant strategy to search as much as possible in the second period. Therefore in any SPE, $I_{1}+I_{2}=x$. Consider, for instance, an asymmetric equilibrium with player 1 searching $\beta_{1}=2 / 3$ of the remaining unsearched area and player 2 searching the remainder, so that $\beta_{2}=1 / 3$. The one-period value for player $i$ in the subgame with the unsearched area of $x$ is

$$
V_{i}(x)=\beta_{i}(1-x) .
$$

Now consider the first period. Note that if player $i$ searches $\tilde{I}_{i}$ in the first period, his expected payoff from two-period game is

$$
\tilde{I}_{i} / x_{1}-\tilde{I}_{i}+\delta\left(1-\left(\tilde{I}_{1}+\tilde{I}_{2}\right) / x_{1}\right) V_{i}\left(x_{1}-\tilde{I}_{1}-\tilde{I}_{2}\right),
$$

where player $i$ incurs search cost of $\tilde{I}_{i}$, gets immediate expected payoff of $\tilde{I}_{i} / x_{1}$, and anticipates the expected payoff of $\delta\left(1-\left(\tilde{I}_{1}+\tilde{I}_{2}\right) / x_{1}\right) V_{i}\left(x_{1}-\tilde{I}_{1}-\tilde{I}_{2}\right)$ in the second period. The first-order conditions with respect to $\tilde{I}_{i}$ are

$$
\tilde{I}_{1}+\tilde{I}_{2}=\frac{1-x_{1}}{2 \delta \beta_{i}}+x_{1}-0.5
$$

In the case of the first player, the preferred combined search in the first period is $\tilde{I}_{1}+\tilde{I}_{2}=0.5$. In the case of the second player, the preferred combined search in the first period is $\tilde{I}_{1}+\tilde{I}_{2}=0.6$. Consequently, there is a unique asymmetric SPE that involves only the second player searching $\tilde{I}_{2}=0.6$ in the first period and both players searching in the second period. The intuition is that as the second player expects a smaller payoff in the second period, he is willing to search more in the first period. The values in the two-period game with the island size of $x_{1}=0.9$ for players 1 and 2 are $\tilde{V}_{1}\left(x_{1}\right)=\frac{7}{60}$ and $\tilde{V}_{2}\left(x_{1}\right)=\frac{1}{8}$, respectively.

\section{References}

[1] Admati, A., and M. Perry (1991): "Joint projects without commitment," Review of Economic Studies, 58, 259-76.

[2] Ahlberg, H., E. Nielson, and J. Walsh (1967): The theory of splines and their applications. New York: Academic Press.

[3] Battaglini, M., S. Nunnari, and T. Palfrey (2014): "Dynamic free riding with irreversible investments," American Economic Review, 104, 2858-2871.

[4] Bhaskar, V., G. J. Mailath and S. Morris (2012): "A foundation for Markov equilibria in infinite horizon perfect information games," PIER Working Paper No. 12-043.

[5] Bonatti, A., and J. Hörner (2011): "Collaborating," American Economic Review, 101, 632663.

[6] Cardon, J., and D. Sasaki (1998): "Preemtive search and R\&D clustering," Rand Journal of economics, 29, 2, 324-338. 
[7] Chatterjee, K., and R. Evans (2004): "Rivals search for buried treasure: competition and duplication in R\&D," Rand Journal of Economics, 35, 160-183.

[8] Che, Y.K., and I. Gale (2003): "Optimal design of research contests," American Economic Review, 93, 646-671.

[9] Choi, J., and H. Gerlach (2014): "Selection biases in complementary R\&D projects," Journal of Economics and Management Strategy, 23(4), 899-924.

[10] Compte, O., and P. Jehiel (2004): "Gradualism in bargaining and contribution games," Review of Economics Studies, 71, 975-1000.

[11] Dasgupta, P., and J. Stiglitz (1980a): "Industrial structure and the nature of innovative activity," The Economic Journal, 90, 266-293.

[12] Dasgupta, P., and J. Stiglitz (1980b): "Uncertainty, industrial structure and the speed of R and D," Bell Journal of Economics, 11, 1-28.

[13] The Economist (1985): "Aircraft industry: the big six: a survey," June 1, 8.

[14] Eichler, H. G., F. Pétavy, F. Pignatti and G. Rasi (2013): "Access to patient-level trial data - a boon for drug developers," New England Journal of Medicine, 369 (17), 1577-9.

[15] Erat, S., and V. Krishnan (2012): "Managing delegated search over design spaces," Management Science, 58, 3, 606-623.

[16] Ericson, R., and A. Pakes (1995): "Markov-perfect industry dynamics: a framework for empirical work," Review of Economics Studies, 62, 53-82.

[17] Fershtman, C., and A. Rubinstein (1997): "A simple model of equilibrium in search procedures," Journal of Economic Theory, 72, 432-441.

[18] Fudenberg, D., and J. Tirole (1985): "Preemption and rent equalization in the adoption of new technology," Review of Economic Studies, 52, 383-401.

[19] Fullerton, R.L., and R. P. McAfee (1999): "Auctioning entry into tournaments," Journal of Political Economy, 107, 573-605.

[20] Georgiadis, G. (2015): "Projects and team dynamics," Review of Economics Studies, 82(1), $187-218$.

[21] Konrad, K. (2014): "Search duplication in research and design spaces - exploring the role of local competition," International Journal of Industrial Organization, 37, 222-228.

[22] Lee, T., and L. L. Wilde (1980): "Market structure and innovation: a reformulation," Quarterly Journal of Economics, 94, 429-436.

[23] Lockwood, B. and J. Thomas (2002): "Gradualism and irreversibility," Review of Economics Studies, 69, 339-356.

[24] Long, NV. (2010): A Survey of Dynamic Games in Economics. Singapore: World Scientific.

[25] Long, NV. (2011): "Dynamic games in the economics of natural resources," Dynamic Games and Applications 1, 115-148. 
[26] Loury, G. C. (1979): "Market structure and innovation," Quarterly Journal of Economics, 93, 395-410.

[27] Mailath, G. and L. Samuelson (2006): Repeated games and reputations: long-run relationships. Oxford University Press.

[28] Marx, L., and S. Matthews (2000): "Dynamic voluntary contribution to a public project," Review of Economics Studies, 67, 327-58.

[29] Maskin, E., and J. Tirole (1988): "A theory of dynamic oligopoly, I: overview and quantaty competition with large fixed costs," Econometrica, 56, 549-569.

[30] Matros, A., and V. Smirnov (2011): "Treasure game," Economics Working Paper Series, University of Sydney, 10.

[31] Matthews, S. (2013): "Achievable outcomes of dynamic contribution games," Theoretical Economics, 8, 365-403.

[32] Merton, R. (1973): "Singletons and multiples in scientific discovery," in R. Merton and N. Storer, eds. The Sociology of Science. Chicago: University of Chicago Press.

[33] Reinganum, J. F. (1981): "Dynamic games of innovation," Journal of Economic Theory, 25, $21-41$.

[34] Reinganum, J. F. (1982): "A dynamic game of R and D: patent protection and competitive behavior," Econometrica, 50, 671-88.

[35] Reinganum, J. F. (1989): "The timing of innovation: research, development, and diffusion," Handbook of Industrial Organization, Vol. 1, 849-908.

[36] Stokey, N., R. Lucas, and E. Prescott (1989): Recursive methods in economic dynamics. Harvard University Press.

[37] Taylor, C. R. (1995): "Digging for golden carrots: an analysis of research tournaments," American Economic Review, 85, 872-890.

[38] Yildirim, H. (2004): "Piecewise Procurement of a Large-Scale Project," International Journal of Industrial Organization, 22, 1349-1375.

[39] Yildirim, H. (2006): "Getting the Ball Rolling: Voluntary Contributions to a Large-Scale Public Project," Journal of Public Economic Theory, 8, 503-528. 